\title{
LOCAL AND GLOBAL SURVIVAL FOR NONHOMOGENEOUS RANDOM WALK SYSTEMS ON $\mathbb{Z}$
}

\author{
DANIELA BERTACCHI, ${ }^{*}$ Università di Milano-Bicocca \\ FÁBIO PRATES MACHADO, ${ }^{* *}$ Universitade de São Paulo \\ FABIO ZUCCA, ${ }^{* * *}$ Politecnico di Milano
}

\begin{abstract}
We study an interacting random walk system on $\mathbb{Z}$ where at time 0 there is an active particle at 0 and one inactive particle on each site $n \geq 1$. Particles become active when hit by another active particle. Once activated, the particle starting at $n$ performs an asymmetric, translation invariant, nearest neighbor random walk with left-jump probability $l_{n}$. We give conditions for global survival, local survival, and infinite activation both in the case where all particles are immortal and in the case where particles have geometrically distributed lifespan (with parameter depending on the starting location of the particle). More precisely, once activated, the particle at $n$ survives at each step with probability $p_{n} \in[0,1]$. In particular, in the immortal case, we prove a $0-1$ law for the probability of local survival when all particles drift to the right. Besides that, we give sufficient conditions for local survival or local extinction when all particles drift to the left. In the mortal case, we provide sufficient conditions for global survival, local survival, and local extinction (which apply to the immortal case with mixed drifts as well). Analysis of explicit examples is provided: we describe completely the phase diagram in the cases $\frac{1}{2}-l_{n} \sim \pm 1 / n^{\alpha}, p_{n}=1$ and $\frac{1}{2}-l_{n} \sim \pm 1 / n^{\alpha}, 1-p_{n} \sim 1 / n^{\beta}$ (where $\alpha, \beta>0$ ).
\end{abstract}

Keywords: Inhomogeneous random walk; frog model; egg model; local survival; global survival

2010 Mathematics Subject Classification: Primary 60K35; 60G50

\section{Introduction}

We study an interacting random walk system on $\mathbb{Z}$ where at time 0 there is one active particle at 0 and one inactive particle at each vertex of $\mathbb{N} \backslash\{0\}=\{1,2, \ldots\}$ (our results apply also if at time 0 there are empty vertices in $\mathbb{N} \backslash\{0\}$; see Section 3 ). Particles become active if an active particle jumps to their location. The behavior of the system depends on two sequences $\left\{l_{n}\right\}_{n \geq 0}$ and $\left\{p_{n}\right\}_{n \geq 0}$ of numbers in $(0,1)$ and $[0,1]$, respectively. The particle which at time 0 was at $n$, once activated, has a geometrically distributed lifespan with parameter $1-p_{n}$ and while alive performs a nearest neighbor random walk with probability $l_{n}$ of jumping to the left and $1-l_{n}$ of jumping to the right. If $p_{n}=1$ we say that the particle is immortal, otherwise it is mortal. We are interested in establishing, depending on the parameters, whether the process survives

\footnotetext{
Received 2 January 2013; revision received 20 March 2013.

* Postal address: Dipartimento di Matematica e Applicazioni, Università di Milano-Bicocca, Via Cozzi 53, 20125 Milano, Italy. Email address: daniela.bertacchi@unimib.it

** Postal address: Instituto de Matemática e Estatística, Universitade de São Paulo, Rua do Matão 1010, São Paulo, Brasil. Email address: fmachado@ime.usp.br

*** Postal address: Dipartimento di Matematica, Politecnico di Milano, Piazza Leonardo da Vinci 32, 20133 Milano, Italy. Email address: fabio.zucca@polimi.it
} 
globally, survives locally, and if there is infinite activation or not. Local and global survival have been studied for several processes; among these it is worth mentioning the contact process and the branching random walks in continuous and discrete time (see, for instance, [3], [4], [5], [6], [12], [13], [14], and [18]).

To be precise, if $L_{0}$ is the event that site 0 is visited infinitely many times (by the same particle or by different particles), we say that there is local survival if $L_{0}$ has positive probability and almost sure local survival if $L_{0}$ has probability 1 . When there is no local survival, that is, when $L_{0}$ has probability 0 , we also say that there is local extinction. We say that there is global survival if, with positive probability, at any time there is at least one active particle, and we say that there is infinite activation if, with positive probability, at arbitrarily large times there are particles which turn from inactive to active.

This process can be seen as a model for information or disease spreading: every active particle has some information and it shares that information with all particles it encounters on its way. In the last decade, different versions of this model have been studied, often under the name frog model or egg model. In [17] the authors proved almost sure local survival for a system of simple random walkers on $\mathbb{Z}^{d}$. This result was extended in [15] to the case of a random initial configuration $(d \geq 3)$ and in [8] for random walks on $\mathbb{Z}$ with right drift. Shape theorems on $\mathbb{Z}^{d}$ can be found in [1] and [2]. Phase transitions for the model where particles have a $g(1-p)$-distributed lifespan, were investigated in [1], [7], [11], and [16]. Recently, in [10], global survival of an asymmetric inhomogeneous random walk system on $\mathbb{Z}$ was studied (in that model particles die after $L$ steps without activation). The present paper is a partial extension of [8] and [10] since we study local and global survival of an inhomogeneous system of random walkers where the initial configuration is given by one particle per site with geometric lifespans $\left\{g\left(1-p_{n}\right)\right\}_{n \in \mathbb{N}}$. This covers the case of an inhomogeneous initial configuration with 0 or 1 particles at site $n$ (if $p_{n}>0$ or $p_{n}=0$, respectively). For instance, Theorem 2.1, below, is an extension of [8, Theorem 2.2] in the case of a deterministic initial configuration with 0 or 1 particle per site (as explained at the beginning of Section 4).

Here is a sketch of the formal construction of our process. Let $(\Omega, \mathcal{F}, \mathbb{P})$ be a probability space and $\left\{\left\{Z_{n}^{i}\right\}_{n \in \mathbb{N}}\right\}_{i \in \mathbb{N}}$ a family of independent random walks on $\mathbb{N} \cup\{D\}$ (where $D \notin \mathbb{N}$ is an absorbing state that we call death state) such that $\left\{Z_{n}^{i}\right\}_{n \in \mathbb{N}}$ starts from $i$ (that is, $Z_{0}^{i}=i$ ). At each step, if the $i$ th walker is not at $D$, it jumps to the left with probability $p_{i} l_{i}$, to the right with probability $p_{i}\left(1-l_{i}\right)$, and to $D$ with probability $1-p_{i}$. Once in $D$ the walkers stay there indefinitely with probability 1 . If $p_{i}=1$ then the $i$ th walker is immortal, while if $p_{i}=0$ then the $i$ th walker goes to $D$ immediately (it is like having no particle at $i$ since it does not participate in the evolution). Our frog model is a collection of dependent walks $\left\{\left\{X_{n}^{i}\right\}_{n \in \mathbb{N}}\right\}_{i \in \mathbb{N}}$ constructed iteratively as follows. Let $X_{n}^{0}=Z_{n}^{0}=0$ for all $n \in N$. Suppose that we have defined $\left\{\left\{X_{n}^{i}\right\}_{n \in \mathbb{N}}\right\}_{i \leq N}$; let us define $\left\{X_{n}^{N+1}\right\}_{n}$. Let $T_{N+1}=\min \{k$ : there exists $i \in$ $\left.\{0, \ldots, N\}, X_{k}^{i}=N+1\right\}($ where $\min (\varnothing):=+\infty)$. Then, for all $\omega \in \Omega$,

$$
X_{n}^{N+1}(\omega):= \begin{cases}N+1, & n<T_{N+1}(\omega) \leq \infty, \\ Z_{n-T_{N+1}^{N}(\omega)}^{N+1}(\omega), & T_{N+1}(\omega) \leq n<\infty .\end{cases}
$$

Here is the outline of the paper and of its main results. We first deal, in Section 2, with the case where all particles are immortal (that is, $p_{n}=1$ for all $n \geq 0$ ). It is obvious that in this case there is always global survival, but infinite activation is trivial only in the case where at least one particle has $l_{n} \leq \frac{1}{2}$. Local survival is nontrivial unless $l_{n}=\frac{1}{2}$ for some $n \in \mathbb{N}$. In order to understand what the difficulties we encounter are, think of the case where all particles drift to the right (we refer to this situation as the right-drift case): infinite activation is guaranteed but local 
survival is not. Theorem 2.1(a) states that, in this case, the probability of local survival obeys a $0-1$ law. Roughly speaking (see Corollary 2.1) in the right-drift case, if $l_{n} \uparrow \frac{1}{2}$ sufficiently fast, then we have almost sure local survival, otherwise we have local extinction. Corollary 2.1 gives conditions which quantify how fast the convergence to $\frac{1}{2}$ should be in order to ensure local survival. On the other hand, if all particles drift to the left (left-drift case), local survival and infinite activation have the same probability (Theorem 2.1(b)). Proposition 2.1 and Remark 2.2 provide sufficient conditions for infinite activation (thus also for local survival) in the left drift case. The idea in Remark 2.2 is that with positive probability there is a simple 'chain reaction' where the initial particle visits a certain site to its right, then the particle there visits a certain site to its right, and so on. Example 2.3 shows that the fact that every possible chain reaction has probability 0 is not sufficient to ensure almost sure finite activation (thus, Proposition 2.1 is indeed a stronger result). Theorem 2.2 states that if certain subsequences of $\left\{l_{n}-\frac{1}{2}\right\}_{n \geq 0}$ (when $l_{n}>\frac{1}{2}$ ) and of $\left\{n\left(\frac{1}{2}-l_{n}\right)\right\}_{n \geq 0}$ (when $l_{n}<\frac{1}{2}$ ) stay in some $\ell^{p}$ space, then there is local survival. By Proposition 2.2, if $\inf _{n \in \mathbb{N}} l_{n}>\frac{1}{2}$ then there is no infinite activation (thus no local survival). Examples 2.2 and 2.4 show that if $\inf _{n \in \mathbb{N}} l_{n}=\frac{1}{2}$ nothing can a priori be said about infinite activation. Examples 2.1 and 2.2 together describe completely the phase diagram of the immortal particle model where $\frac{1}{2}-l_{n} \sim \pm 1 / n^{\alpha}, \alpha>0$.

Section 3 is devoted to the case where each particle may be mortal and has geometrical lifespan with parameter $1-p_{n}, p_{n} \in[0,1]$. If $p_{n}<1$ for all $n$ then any particle disappears almost surely after a finite number of steps; thus, global survival is no longer guaranteed and, even if all particles have right drift, neither is infinite activation. Indeed, in this case global survival and infinite activation have the same probability. In Section 3.1 we give sufficient conditions for global extinction (Proposition 3.1) and for global survival (Theorem 3.1). In particular, we show that to survive globally it is necessary that $\lim \sup _{n} p_{n}=1$ and it is sufficient that certain subsequences of $\left\{1-p_{n}\right\}_{n \geq 0}$ and of $\left\{l_{n}-\frac{1}{2}\right\}_{n \geq 0}$ stay in some $\ell^{p}$ space. In Section 3.2 we deal with the problem of local survival of the process and give some sufficient conditions on the speed of decay of $\left\{1-p_{n}\right\}_{n \geq 0}$ and of $\left\{l_{n}-\frac{1}{2}\right\}_{n \geq 0}$ which imply local extinction (Theorem 3.2) or local survival (Theorem 3.3). Corollary 3.1 shows how our results apply to the case $1-p_{n} \sim 1 / n^{\beta}(\beta>0)$ and $\frac{1}{2}-l_{n} \sim \pm 1 / n^{\alpha}(\alpha>0)$, completely describing the phase diagrams in these cases.

All the proofs are to be found in Section 5, while in Section 4 we comment on some further questions which could be investigated: one possible generalization is the study of the process in random environments (see Theorem 4.1).

\section{Immortal particles}

In this section, all particles are immortal, that is $p_{n}=1$ for all $n \geq 0$. This assumption guarantees global survival, nevertheless local survival and infinite activation need additional conditions on the sequence $\left\{l_{n}\right\}_{n \geq 0}$. Clearly, if for some $n \in \mathbb{N}, l_{n}=\frac{1}{2}$ then there is local survival and infinite activation (with positive probability the initial particle reaches $n$ and the random walk associated with $n$ is recurrent). Therefore, in this section we assume that $l_{n} \neq \frac{1}{2}$ for all $n$.

Let $A_{n}$ be the event that the particle at $n$ is activated and ever visits 0 and $B_{n}$ the event that the particle at $n$ is activated sooner or later. Clearly $A_{n} \subseteq B_{n}$ and $\mathbb{P}\left(A_{n}\right)>0$ for all $n \in \mathbb{N}$ (since $l_{n} \in(0,1)$ for all $\left.n \in \mathbb{N}\right)$. Note that $\left\{A_{n}\right.$ i.o. $\} \subseteq L_{0}$ and $\mathbb{P}\left(L_{0} \backslash\left\{A_{n}\right.\right.$ i.o. $\left.\}\right)=0$. (Here, i.o. denotes infinitely often.) Moreover if there exists $n$ such that $l_{n}<\frac{1}{2}$ then $\mathbb{P}\left(B_{m} \mid B_{n}\right)=1$ for all $m>n$; thus, in this case there is infinite activation. 
For any choice of $\left\{l_{n}\right\}_{n \geq 0}$, by standard random walk computations, we have

$$
\mathbb{P}\left(A_{n} \mid B_{n}\right)= \begin{cases}1 & \text { if } l_{n}>\frac{1}{2}, \\ \left(\frac{l_{n}}{1-l_{n}}\right)^{n} & \text { if } l_{n}<\frac{1}{2} .\end{cases}
$$

Let $B_{\infty}=\bigcap_{n=1}^{\infty} B_{n}$ be the event that all the particles are activated sooner or later; $B_{\infty}$ represents infinite activation. Our first goal is to find conditions on the sequence $\left\{l_{n}\right\}_{n \geq 0}$ which guarantee local survival. The knowledge of the behaviour of the system with a fixed sequence helps to characterize many other sequences. Namely, by coupling it is not difficult to show that in the right-drift case if we have local survival with $\left\{l_{n}\right\}_{n \geq 0}$, then there is local survival with any $\left\{l_{n}^{\prime}\right\}_{n \geq 0}$ such that $l_{n}^{\prime} \geq l_{n}$ for all $n$. Conversely, in the left-drift case, if we have local survival with $\left\{l_{n}\right\}_{n \geq 0}$, then there is local survival with any $\left\{l_{n}^{\prime}\right\}_{n \geq 0}$ such that $l_{n}^{\prime} \leq l_{n}$ for all $n$.

The following theorem includes the particular case of [8, Theorem 2.2] when the initial condition is one particle per site almost surely (there $l_{n}=1-p$ for all $n$ ). This theorem characterizes the right-drift case in terms of the sequence $\left\{l_{n}\right\}_{n \geq 0}$ and shows that in the left-drift case the probability of local survival is equal to the probability of infinite activation.

Theorem 2.1. (a) Suppose that $l_{n}<\frac{1}{2}$ for all $n$ (right-drift case). The probability of local survival obeys a $0-1$ law:

$$
\mathbb{P}\left(A_{n} \text { i.o. }\right)= \begin{cases}0 & \text { if } \sum_{n \in \mathbb{N}}\left(l_{n} /\left(1-l_{n}\right)\right)^{n}<+\infty \\ 1 & \text { otherwise. }\end{cases}
$$

(b) Suppose that $l_{n}>\frac{1}{2}$ for all $n$ (left-drift case). Then $\mathbb{P}\left(B_{\infty} \triangle\left(A_{n}\right.\right.$ i.o. $\left.)\right)=0$ (where $\triangle$ denotes symmetric difference of sets).

The following corollary gives some conditions which are easy to check and that imply convergence or divergence of the characterizing series of Theorem 2.1(a). We denote the natural logarithm by $\ln (\cdot)$.

Corollary 2.1. In the right-drift case $\left(l_{n}<\frac{1}{2}\right.$ for all $\left.n\right)$, the following statements hold.

(a) If $\lim \inf _{n} n\left(\frac{1}{2}-l_{n}\right)<+\infty$ then $\mathbb{P}\left(A_{n}\right.$ i.o. $)=1$.

(b) If

$$
\frac{n\left(\frac{1}{2}-l_{n}\right)}{\ln (n)} \leq \frac{1+\ln (\ln (n)) / \ln (n)}{4-2(\ln (n)+\ln (\ln (n))) / n}
$$

for every sufficiently large $n$, then $\mathbb{P}\left(A_{n}\right.$ i.o. $)=1$.

(c) If

$$
\frac{n\left(\frac{1}{2}-l_{n}\right)}{\ln (n)} \geq \frac{1+\beta \ln (\ln (n)) / \ln (n)}{4-2(\ln (n)+\beta \ln (\ln (n))) / n}
$$

for some $\beta>1$ and every sufficiently large $n$, then $\mathbb{P}\left(A_{n}\right.$ i.o. $)=0$.

(d) If there exists $\lambda<4$ such that $\sum_{n} \exp \left(-\lambda n\left(\frac{1}{2}-l_{n}\right)\right)<+\infty$ then $\mathbb{P}\left(A_{n}\right.$ i.o. $)=0$.

The previous result implies that, even if $l_{n} \uparrow \frac{1}{2}$, there is no local survival if $l_{n}$ converges to $\frac{1}{2}$ too slowly. Moreover, when $\lim _{n} n\left(\frac{1}{2}-l_{n}\right) / \ln (n)$ exists then there is a critical threshold, 
namely $\frac{1}{4}$, separating local survival from local extinction. More precisely, if $\lim _{n} n\left(\frac{1}{2}-\right.$ $\left.l_{n}\right) / \ln (n)<\frac{1}{4}$ then there is local survival (see Corollary 2.1(b)), while if $\lim _{n} n\left(\frac{1}{2}-l_{n}\right) / \ln (n)>$ $\frac{1}{4}$ then there is extinction (see Corollary 2.1(c)). Finally, if $\lim _{n} n\left(\frac{1}{2}-l_{n}\right) / \ln (n)=\frac{1}{4}^{-}$(that is, the sequence converges from below) then there is local survival again (see Corollary 2.1(b)). As for the behavior when $\lim _{n} n\left(\frac{1}{2}-l_{n}\right) / \ln (n)=\frac{1}{4}^{+}$we can have either local survival or local extinction: indeed, if the equality in Corollary 2.1(b) holds then we have local survival, while if the equality in Corollary 2.1(c) holds we have local extinction.

It is easy to extend Theorem 2.1 to the cases where there are both particles with right drift and particles with left drift, as we note in the following remark, which allows us to focus only on the two 'pure' cases where all particles drift towards the same direction.

Remark 2.1. If all but a finite number of particles have right drift then, by Theorem 2.1(a), the convergence $\sum_{n: l_{n}<1 / 2}\left(l_{n} /\left(1-l_{n}\right)\right)^{n}<+\infty$ implies local extinction. If the series diverges, then $\mathbb{P}\left(A_{n}\right.$ i.o. $)=\mathbb{P}\left(B_{\infty}\right)=\mathbb{P}\left(B_{j}\right)$, where $j=\min \left\{n: l_{n}<\frac{1}{2}\right\}$; hence, we have local survival, since $\mathbb{P}\left(B_{j}\right)>0$ (to prove that $\mathbb{P}\left(A_{n}\right.$ i.o. $\left.\mid B_{j}\right)=1$ when the series diverges, we have to mimic the proof of Theorem 2.1(a)).

On the other hand, if there is an infinite number of particles with left drift and at least one with right drift, then again we have local survival, since $\mathbb{P}\left(A_{n}\right.$ i.o. $)=\mathbb{P}\left(B_{j}\right)$, where $j=\min \left\{n: l_{n}<\frac{1}{2}\right\}$.

The Remark 2.1 and Corollary 2.1 are useful for the analysis of the following example.

Example 2.1. Let $\frac{1}{2}-l_{n} \sim 1 / n^{\alpha}$ as $n \rightarrow \infty$ (where $\alpha>0$ ). Since $l_{n}<\frac{1}{2}$ for every sufficiently large $n \in \mathbb{N}$, by Remark 2.1 it is enough to consider the case $l_{n}<\frac{1}{2}$ for all $n \in \mathbb{N}$. It is clear that if $\alpha \geq 1$ then $n\left(\frac{1}{2}-l_{n}\right) / \ln (n) \rightarrow 0$; thus, by Corollary 2.1(b), there is local survival. Conversely, if $\alpha \in(0,1)$ then $n\left(\frac{1}{2}-l_{n}\right) / \ln (n) \rightarrow+\infty$; hence, there is local extinction according to Corollary 2.1(c).

In the left-drift case, Theorem 2.1(b) tells us that local survival and infinite activation have the same probability. Thus, it is interesting to find conditions for $\mathbb{P}\left(B_{\infty}\right)>0$. The first result is the following proposition; its proof makes use of a coupling between the frog model and the rumor process (see [9] for the formal definition).

Proposition 2.1. Suppose that $l_{n}>\frac{1}{2}$ for all $n \geq 0$. If, for some increasing sequence $\left\{n_{k}\right\}_{k \in \mathbb{N}}$,

$$
\sum_{k=0}^{\infty} \prod_{i=0}^{n_{k}}\left(1-\left(\frac{1-l_{i}}{l_{i}}\right)^{n_{k+1}-i}\right)<+\infty
$$

then $\mathbb{P}\left(B_{\infty}\right)>0$.

The main idea in the proof of Proposition 2.1 is to provide a positive lower bound for the probability that, for each $k$, at least one particle between 0 and $n_{k}$ visits site $n_{k+1}$. These events, indexed by $k$, are not independent since they involve overlapping sets of particles.

If we consider disjoint sets of particles, we still have dependence of the associated walks $\left\{\left\{X_{n}^{i}\right\}_{n \in \mathbb{N}}\right\}_{i \in \mathbb{N}}$, since, to start moving, each particle needs to be activated by another walker. Nevertheless, disjoint sets $I$ and $J$ have corresponding walks $\left\{\left\{Z_{n}^{i}\right\}_{n \in \mathbb{N}}\right\}_{i \in I}$ and $\left\{\left\{Z_{n}^{i}\right\}_{n \in \mathbb{N}}\right\}_{i \in J}$ which are independent by construction. This motivates the following strategy, used throughout the whole paper. We choose a sequence of nonvoid, pairwise disjoint sets $\left\{\mathscr{B}_{n}\right\}_{n \in \mathbb{N}}, \mathscr{B}_{n} \subset \mathbb{N}$, that we call blocks. The general idea is to estimate the probability that particles in different blocks perform specific different tasks. One way of choosing the sequence $\left\{\mathscr{B}_{n}\right\}_{n \in \mathbb{N}}$ is to 


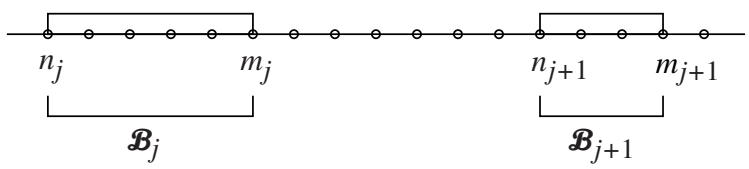

Figure 1.

partition $\mathbb{N}$ into disjoint intervals and to choose, for every $n \in \mathbb{N}, \mathcal{B}_{n}$ as a subset of the $n$th interval (as in Figure 1).

As an example, consider, for each $j \geq 0$, the event $B_{j}^{\prime}:=$ 'at least one particle in $\mathcal{B}_{j}$ visits all the sites of $\mathcal{B}_{j+1}$ '. Using the explicit construction of the process, it is easy to show that $\bigcap_{j \in \mathbb{N}} B_{j}^{\prime}$ is the same event if we consider the frog model $\left\{\left\{X_{n}^{i}\right\}_{n \in \mathbb{N}}\right\}_{i \in \mathbb{N}}$ or the process $\left\{\left\{Z_{n}^{i}\right\}_{n \in \mathbb{N}}\right\}_{i \in \mathbb{N}}$. Using the second process, however, allows us to take advantage of the fact that we are dealing with an intersection of independent events. Clearly, $\bigcap_{j \in \mathbb{N}} B_{j}^{\prime}$ is a subset of $B_{\infty}$; thus, if it has positive probability, then there is infinite activation, which, in the left-drift case, also means local survival. The probability of $\bigcap_{j \in \mathbb{N}} B_{j}^{\prime}$ is strictly positive if and only if $\sum_{j=0}^{\infty} \prod_{i \in \mathcal{B}_{j}}\left(1-\left(\left(1-l_{i}\right) / l_{i}\right)^{m_{j+1}-i}\right)<+\infty$, where $m_{j}:=\max \left(\mathscr{B}_{j}\right)$ (the proof mimics the one of Proposition 2.1); hence, the previous inequality implies $\mathbb{P}\left(B_{\infty}\right)>0$. In this case, the block decomposition simply gives us a corollary of Proposition 2.1; in what follows we use this method to obtain sufficient conditions for local and global survival in the general case.

A special case of block decomposition is given by $\mathcal{B}_{j}:=\left\{n_{j}\right\}$ for a suitable increasing sequence $\left\{n_{j}\right\}_{j \in \mathbb{N}}$ and it is treated in the following remark.

Remark 2.2. Suppose that all particles have left drift $\left(l_{n}>\frac{1}{2}\right.$ for all $\left.n\right)$. If there exists an increasing sequence $\left\{n_{j}\right\}_{j \in \mathbb{N}}$ such that $\prod_{j \in \mathbb{N}}\left(\left(1-l_{j}\right) / l_{j}\right)^{n_{j+1}-n_{j}}>0$ or, equivalently,

$$
\sum_{j=0}^{\infty}\left(n_{j+1}-n_{j}\right) \frac{2 l_{n_{j}}-1}{l_{n_{j}}}<+\infty,
$$

then $\mathbb{P}\left(B_{\infty}\right)>0$. To be precise, we are exploiting the fact that if there exists a subsequence $\left\{n_{j}\right\}_{j \geq 0}$ in $\mathbb{N}$, such that the event 'the $n_{j}$ th particle visits the $n_{j+1}$ th vertex, for all $j \geq 0$ ' has positive probability, then $B_{\infty}$ also has positive probability.

We may think that we need to test infinitely many sequences looking for the one satisfying (2.2). Actually, there is one particular sequence $\left\{\bar{n}_{j}\right\}_{j \in \mathbb{N}}$ such that if either it is finite or it is infinite but does not satisfy (2.2), then there are no sequences satisfying (2.2). Define the increasing sequence $\left\{\bar{n}_{j}\right\}_{j \in \mathbb{N}}$ as the collection of all points satisfying $h(n)<h(n-1)$, where $h(n):=\min \left\{\left(2 l_{k}-1\right) / l_{k}: k \leq n\right\}$. The claim follows from Lemma 5.3, below. In particular, if $\left\{l_{n}\right\}_{n \geq 0}$ is decreasing then $\bar{n}_{j}=j$ for all $j \in \mathbb{N}$.

Finally, there might be infinite activation even if there are no sequences satisfying (2.2), as Example 2.3, below, shows.

For all $k \geq 0$, the event 'the $n_{k}$ th particle visits the $n_{k+1}$ th vertex' implies the event 'at least one particle between 0 and $n_{k}$ visits the $n_{k+1}$ th vertex'; hence, (2.2) implies (2.1). Nevertheless, the condition given by Remark 2.2 is easier to check.

The block argument leads to other nice sufficient conditions for local survival. The following result is a particular case of Theorem 3.3(c), below, when $p_{n}=1$ for all $n \in \mathbb{N}$ and it deals simultaneously with the mixed case.

Theorem 2.2. Suppose that there exists $L \in \mathbb{N}$ and a sequence of pairwise disjoint sets $\left\{\mathcal{B}_{n}\right\}_{n \in \mathbb{N}}$

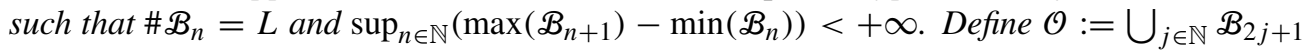


and $\mathscr{E}:=\bigcup_{j \in \mathbb{N}} \mathcal{B}_{2 j}$. If

$$
\sum_{n \in \mathcal{E}: l_{n}>1 / 2}\left(l_{n}-\frac{1}{2}\right)^{L}<+\infty, \quad \sum_{n \in \mathcal{O}: l_{n}<1 / 2} n^{L}\left(\frac{1}{2}-l_{n}\right)^{L}<+\infty,
$$

then there is local survival.

In Theorem 2.2, the idea is to make sure that particles in even labeled blocks $\&$ take care of the activation process while particles in odd labeled blocks $\mathcal{O}$ take care of visiting the origin (at least one particle for each block).

In particular, we have that a sufficient condition for local survival in the left-drift case (and, respectively, the right-drift case) is the existence of $L, m \in \mathbb{N} \backslash\{0\}$ and of an increasing sequence $\left\{n_{j}\right\}_{j \in \mathbb{N}}, n_{j+1}-n_{j} \leq m$, such that

$$
\sum_{j \geq 0}\left(l_{n_{j}}-\frac{1}{2}\right)^{L}<+\infty, \quad\left(\text { respectively, } \sum_{j \geq 0} n_{j}{ }^{L}\left(\frac{1}{2}-l_{n_{j}}\right)^{L}<+\infty\right) .
$$

Indeed, if we take $L_{1}=L m$, then every interval $\left[n L_{1},(n+1) L_{1}-1\right]$ contains at least $L$ vertices in $\left\{n_{j}\right\}_{j \in \mathbb{N}}$. For all $n \in \mathbb{N}$, take exactly $L$ of these vertices in the $n$th interval and define $\mathcal{B}_{n}$ as the set containing those vertices. Theorem 2.2 yields the conclusion. Other, more powerful, conditions can be derived from Theorem 3.3(b) and (c), below, by taking $p_{n}=1$ for all $n \in \mathbb{N}$.

Here are two examples which make use of Theorem 2.2. The first one is the left-drift counterpart of Example 2.1.

Example 2.2. Take $l_{n}-\frac{1}{2} \sim 1 / n^{\alpha}$ as $n \rightarrow \infty$ where $\alpha>0$; hence, $l_{n}>\frac{1}{2}$ for every sufficiently large $n \in \mathbb{N}$ and if $L>1 / \alpha$ then $\sum_{n}\left(l_{n}-\frac{1}{2}\right)^{L}<\infty$. Thus, by Theorem 2.2, we have local survival and $\mathbb{P}\left(B_{\infty}\right)>0$ for all $\alpha>0$.

The second example shows that the sufficient condition given in Remark 2.2 is not necessary.

Example 2.3. Let $l_{0}=\frac{1}{2}+\varepsilon, \varepsilon \in\left(0, \frac{1}{2}\right)$, and $l_{n}=\frac{1}{2}+1 / j^{2}$ if $j^{3} \leq n<(j+1)^{3}$. By Theorem 2.2 there is local survival, indeed $\sum_{n \in \mathbb{N}}\left(l_{n}-\frac{1}{2}\right)^{L}=\varepsilon^{L}+\sum_{j \geq 1}\left(3 j^{2}+3 j+1\right) / j^{2 L}$ which converges for $L$ sufficiently large. On the other hand, $\left\{l_{n}\right\}_{n \in \mathbb{N}}$ is nonincreasing; hence, by Remark 2.2 and Lemma 5.3, below, there exists a sequence $\left\{n_{j}\right\}_{j \in \mathbb{N}}$ satisfying (2.2) if and only if $\sum_{n \geq 0}\left(l_{n}-\frac{1}{2}\right) / l_{n}<\infty$ : this is false since

$$
\sum_{n \geq 0} \frac{l_{n}-\frac{1}{2}}{l_{n}} \geq \sum_{j \geq 1}\left(3 j^{2}+3 j+1\right) \frac{1 / j^{2}}{\frac{1}{2}+1 / j^{2}}=\infty .
$$

So far, in the left-drift case, we have seen only sufficient conditions for $\mathbb{P}\left(B_{\infty}\right)>0$. We now give a sufficient condition for $\mathbb{P}\left(B_{\infty}\right)=0$, whose proof makes use of a random walk approach.

Proposition 2.2. In the left-drift case $\left(l_{n}>\frac{1}{2}\right.$ for all $\left.n\right)$, if $\operatorname{lim~inf}_{n \rightarrow \infty} l_{n}>\frac{1}{2}$ then $\mathbb{P}\left(B_{\infty}\right)=0$ and there is local extinction.

In the left-drift case, if $\inf _{n \in \mathbb{N}} l_{n}=\frac{1}{2}$ then both $\mathbb{P}\left(B_{\infty}\right)>0$ or $\mathbb{P}\left(B_{\infty}\right)=0$ are possible. Indeed, Example 2.2 shows that survival is possible, while extinction is shown by the following example where $l_{n} \downarrow \frac{1}{2}$ slowly enough such that $\mathbb{P}\left(B_{\infty}\right)=0$. 
Example 2.4. Consider two decreasing sequences $\left\{q_{i}\right\}_{i \in \mathbb{N}}$ and $\left\{\delta_{i}\right\}_{i \in \mathbb{N}}$ such that $q_{i} \downarrow \frac{1}{2}$ and $\delta_{i} \downarrow 0$. Let $n_{0}=0$, we construct $\left\{n_{k}\right\}_{k \in \mathbb{N}}$ iteratively. Suppose that we have defined $n_{i}$ for all $i \leq k$. Let $\mathcal{M}_{k}$ be the random walk system with left-jump probabilities $\left\{\widehat{l_{i}}(k)\right\}_{i \in \mathbb{N}}$ where

$$
\widehat{l_{i}}(k)= \begin{cases}q_{j} & \text { if } j \in\left\{n_{j}, \ldots, n_{j+1}-1\right\}, \text { for all } j<k, \\ q_{k} & \text { if } i \geq n_{k} .\end{cases}
$$

We know that, since $\inf _{i} \widehat{l_{i}}(k)>\frac{1}{2}$, by Proposition 2.2 , almost surely for the model $\mathcal{M}_{k}$ we will have only a finite number of activations. Hence, it is possible to find $n_{k+1}$ large enough such that, with probability at least $1-\delta_{k+1}$, no particles in $\left\{i: i \geq n_{k+1}\right\}$ will be activated in the model $\mathcal{M}_{k}$.

Now, for all $j \in \mathbb{N}$, define $l_{j}=q_{k}$ if $j \in\left\{n_{k}, \ldots, n_{k+1}-1\right\}$ and denote by $\mathcal{M}$ the corresponding random walk system. Clearly, $l_{n} \downarrow \frac{1}{2}$. For the model $\mathcal{M}$, the probability of activating particles in the $(k+1)$ th block is at most $\delta_{k+1}$ (for all $k$ ). This is due to the fact that, since $l_{i}=\widehat{l_{i}}(k)$ for all $i<n_{k+1}$, before the activation of the $n_{k+1}$ th particle, $\mathcal{M}$ and $\mathcal{M}_{k}$ have the same behavior. Hence, with probability 1, sooner or later there will be no new activations and $\mathbb{P}\left(B_{\infty}\right)=0$.

\section{Particles with geometrical lifespan}

We now suppose that the particle at $n$ survives, at each step, with probability $p_{n}$, thus, once activated, it has a lifespan which is $g\left(1-p_{n}\right)$-distributed. More precisely, the probability that the lifetime of the $n$th particle equals $k \geq 1$ is $p_{n}^{k-1}\left(1-p_{n}\right)$ if $p_{n} \in[0,1)$ and 0 if $p_{n}=1$ (in this last case the lifetime is infinite almost surely). The main differences from the immortal particle case are that here global survival is not guaranteed (but it has the same probability as the event of infinite activations) and that the knowledge of the drift, a priori, plays a minor role. Indeed, particles with right drift will activate a finite number of sites almost surely and particles with left drift have probability strictly smaller than 1 of visiting the origin. We assume in this whole section that $p_{n} \in[0,1]$ for all $n$, that is, that every particle can be mortal as well as immortal (clearly $p_{0}>0$ otherwise the process would not start at all). Observe that if $p_{n}=0$ for some $n \in \mathbb{N}$ then those particles do not participate in the evolution of the system; therefore, it is like having a system with empty vertices. On the other hand, if $p_{n}=1$ for some $n$, then there is global survival, since there is a positive probability of activating those particles. We also assume that $l_{n} \in(0,1)$ for all $n \in \mathbb{N}$ and $l_{n} \neq \frac{1}{2}$ for all $n$ such that $p_{n}=1$.

Remark 3.1. By using a coupling between the mortal process and a process with immortal particles with the same sequence $\left\{l_{n}\right\}_{n \in \mathbb{N}}$, it is clear that all sufficient conditions for global or local extinction given in Section 2 extend to the mortal case (compare Examples 2.1 and 2.2 and Corollary 3.1, below). Indeed, the probability of local (or global) survival is nondecreasing with respect to $\left\{p_{n}\right\}_{n \in \mathbb{N}}$. As for the dependence of the probabilities of survival with respect to $\left\{l_{n}\right\}_{n \in \mathbb{N}}$, the discussion before Theorem 2.1 applies. Even in the mortal case the most interesting situations are $\sup l_{n}=\frac{1}{2}$ (if right drift) and $\inf l_{n}=\frac{1}{2}$ (if left drift). Indeed, if $\sup l_{n}<\frac{1}{2}$ then, according to Corollary 2.1, there is local extinction even for an immortal particle system; thus, there is no local survival in the mortal case. If inf $l_{n}>\frac{1}{2}$, by Proposition 2.2, even in the immortal case we activate only a finite number of particles almost surely; thus, there is global extinction in the mortal case. 


\subsection{Conditions for global survival/extinction}

In this case global survival is not trivial unless $p_{n}=1$ for some $n \in \mathbb{N}$. Indeed, if $p_{n}<1$ for all $n \in \mathbb{N}$ then global survival is not guaranteed and has the same probability as $B_{\infty}$, that is, the event of infinite activation. In this case, in order to activate infinitely many sites, we need the action of infinitely many particles. It is no longer true, as it was in the case of immortal particles, that it suffices that there exists a particle with right drift to have infinite activation (that particle would still be activated with positive probability but, if it is mortal, it will almost surely activate only a finite number of particles). The following results give sufficient conditions for global extinction and global survival, respectively.

Proposition 3.1. If $\sup _{n} p_{n}<1$ then $\mathbb{P}\left(B_{\infty}\right)=0$ and there is no global survival almost surely.

Theorem 3.1. If there exists $L \in \mathbb{N}$ and a sequence of pairwise disjoint sets $\left\{\mathscr{B}_{n}\right\}_{n \in \mathbb{N}}$ such that $\# \mathcal{B}_{n}=L, \sup _{n \in \mathbb{N}}\left(\max \left(\mathcal{B}_{n+1}\right)-\min \left(\mathcal{B}_{n}\right)\right)<+\infty$, and

$$
\sum_{k \in \bigcup_{n \in \mathbb{N}} \mathcal{B}_{n}}\left(1-p_{k}\right)^{L / 2}<+\infty, \quad \sum_{k \in \bigcup_{n \in \mathbb{N}} \mathcal{B}_{n}: p_{k} l_{k}>1 / 2}\left(l_{k}-\frac{1}{2}\right)^{L}<+\infty,
$$

then there is global survival.

A sufficient condition for global survival is the existence of $L, m \in \mathbb{N} \backslash\{0\}$ and of an increasing sequence $\left\{n_{j}\right\}_{j \in \mathbb{N}}$, such that $n_{j+1}-n_{j} \leq m, \sum_{j \in \mathbb{N}}\left(1-p_{n_{j}}\right)^{L / 2}<+\infty$, and $\sum_{j: p_{n_{j}} l_{n_{j}}>1 / 2}\left(l_{n_{j}}-\frac{1}{2}\right)^{L}<+\infty$ (see the discussion after Theorem 2.2).

\subsection{Conditions for local survival/extinction}

From now on we deal with local survival and local extinction. The first assertion follows from a Borel-Cantelli argument (as in Theorem 2.1(a)) once we note that

$$
\mathbb{P}\left(A_{n} \mid B_{n}\right)=\left(\frac{1-\sqrt{1-4 p_{n}^{2} l_{n}\left(1-l_{n}\right)}}{2 p_{n}\left(1-l_{n}\right)}\right)^{n},
$$

which follows from random walk computations (see Section 5).

Theorem 3.2. If

$$
\sum_{n}\left(\frac{1-\sqrt{1-4 p_{n}^{2} l_{n}\left(1-l_{n}\right)}}{2 p_{n}\left(1-l_{n}\right)}\right)^{n}<+\infty
$$

then $\mathbb{P}\left(A_{n}\right.$ i.o. $)=0$. In particular, the following statements hold.

(a) Let $\Delta_{n}=1-p_{n}$ and $\delta_{n}=\frac{1}{2}-l_{n}$; if $\delta_{n} \wedge 0 \rightarrow 0$ as $n \rightarrow \infty$ and $\liminf _{n \rightarrow \infty} n\left(2 \delta_{n}+\right.$ $\left.\sqrt{2 \Delta_{n}+4 \delta_{n}^{2}}\right) / \ln (n)>1$ then $\mathbb{P}\left(A_{n}\right.$ i.o. $)=0$.

(b) If $\sum_{n} p_{n}^{n}\left(1-\left(1-2 l_{n}\right)^{+}\right)^{n}<+\infty$ then $\mathbb{P}\left(A_{n}\right.$ i.o. $)=0\left(\right.$ where $\left(1-2 l_{n}\right)^{+}=\left(1-2 l_{n}\right) \vee$ $0)$.

(c) If $\sum_{n} p_{n}^{n}<+\infty$ (for instance, if $\left.\sup _{n} p_{n}<1\right)$ then $\mathbb{P}\left(A_{n}\right.$ i.o. $)=0$ for any choice of $\left\{l_{n}\right\}_{n}$.

The tricky part is finding conditions for local survival: on the one hand we need that all particles get activated sooner or later and on the other hand that infinitely many of them visit the origin. To avoid dealing with situations where a particle is required both to visit a certain number of sites and the origin, we exploit once again the idea of choosing pairwise disjoint 


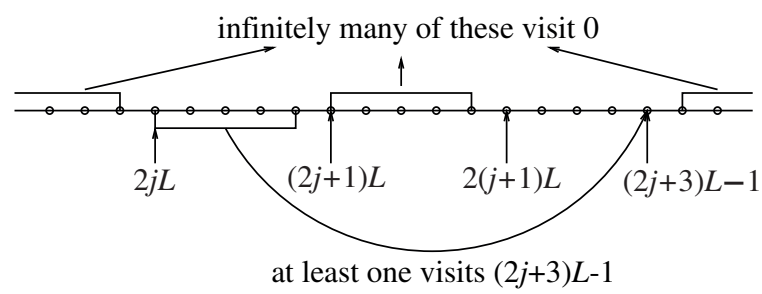

FiguRE 2.

blocks in $\mathbb{N}$. Some blocks will take care of activation and the others of local survival. We choose a sequence of pairwise disjoint sets $\left\{\mathscr{B}_{n}\right\}_{n \in \mathbb{N}}$ of cardinality $L$. The event where, for all $n \in \mathbb{N}$ such that $\max \left(\mathscr{B}_{2 n+2}\right)>\max \left(\mathcal{B}_{2 n}\right)$, at least one of the particles in $\mathscr{B}_{2 n}$ visits the right-most vertex of $\mathscr{B}_{2 n+2}$ and an infinite number of particles in $\bigcup_{n \in \mathbb{N}} \mathscr{B}_{2 n+1}$ visits the origin is a subset of the event of local survival. Thus, a sufficient condition for local survival is that this event has positive probability. In Figure 2 we picture the case $\mathscr{B}_{n}:=[n L,(n+1) L-1]$.

By using (3.1) and the fact that the probability that the $n$th particle, if activated, ever visits the site $m>n$ is

$$
\left(\frac{1-\sqrt{1-4 p_{n}^{2} l_{n}\left(1-l_{n}\right)}}{2 p_{n} l_{n}}\right)^{m-n},
$$

we get a lower bound for the probability of local survival. The main idea in the following theorem is that even labeled blocks take care of infinite activation while odd labeled blocks provide infinitely many particles visiting 0 . In particular, we will suppose that $\left\{\mathcal{B}_{2 n}\right\}_{n \in \mathbb{N}}$ satisfies the conditions in Theorem 3.1 (which guarantees global survival).

Theorem 3.3. Suppose that there exists $L \in \mathbb{N}$ and a sequence of pairwise disjoint sets $\left\{\mathscr{B}_{n}\right\}_{n \in \mathbb{N}}$ such that $\sup _{n \in \mathbb{N}}\left(\max \left(\mathscr{B}_{n+1}\right)-\min \left(\mathscr{B}_{n}\right)\right)<+\infty$ and $\left\{\mathcal{B}_{2 n}\right\}_{n \in \mathbb{N}}$ satisfies the hypotheses of Theorem 3.1. Define $\Delta_{k}:=1-p_{k}, \delta_{k}:=\frac{1}{2}-l_{k}$, and $\mathcal{O}:=\bigcup_{j \in \mathbb{N}} \mathscr{B}_{2 j+1}$. If one of the following conditions holds:

(a) $\liminf \operatorname{in}_{k \rightarrow \infty, k \in \mathcal{O}} k\left(2 \delta_{k}+\sqrt{2 \Delta_{k}+4 \delta_{k}^{2}}\right)<\infty$,

(b) $2 \delta_{k}+\sqrt{2 \Delta_{k}+4 \delta_{k}^{2}} \leq \ln (k) / k$, for all sufficiently large $k \in \mathcal{O}$,

(c) $\sum_{k \in \mathcal{O}}: p_{k}\left(1-l_{k}\right)>1 / 2 k^{L} \delta_{k}^{L}+\sum_{k \in \mathcal{O}} k^{L} \Delta_{k}^{L / 2}<+\infty$,

then there is local survival.

Each of the conditions (a), (b), and (c) of Theorem 3.3 implies the divergence of $\sum_{k \in \mathcal{O}}(1-$ $\left.\sqrt{1-4 p_{k}^{2} l_{k}\left(1-l_{k}\right)}\right)^{k} /\left(2 p_{k}\left(1-l_{k}\right)\right)^{k}$ which, in turn, implies that, conditioned on infinite activation, the probability that an infinite number of particles in $\mathcal{E}$ visits the origin is positive.

Moreover, if we take $\mathcal{B}_{n}=[n L,(n+1) L-1]$, then a sufficient condition for local (and global) survival is $\sum_{n} n^{L}\left(1-p_{n}\right)^{L / 2}<+\infty, \sum_{n: p_{n} l_{n}>1 / 2}\left(l_{n}-\frac{1}{2}\right)^{L}<+\infty$, and $\sum_{n: p_{n}\left(1-l_{n}\right)>1 / 2} n^{L}\left(\frac{1}{2}-l_{n}\right)^{L}<+\infty$.

As a corollary we obtain the complete phase diagram in the case where $\Delta_{k}$ and $\delta_{k}$ decay polynomially (the analogous results in the immortal case have been studied in Examples 2.1 and 2.2). 


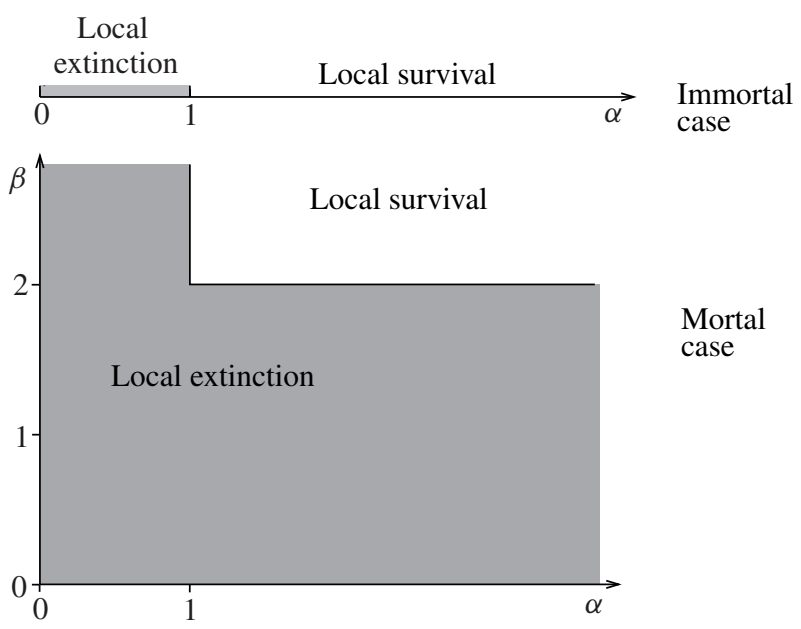

FIGURE 3: The right-drift case.

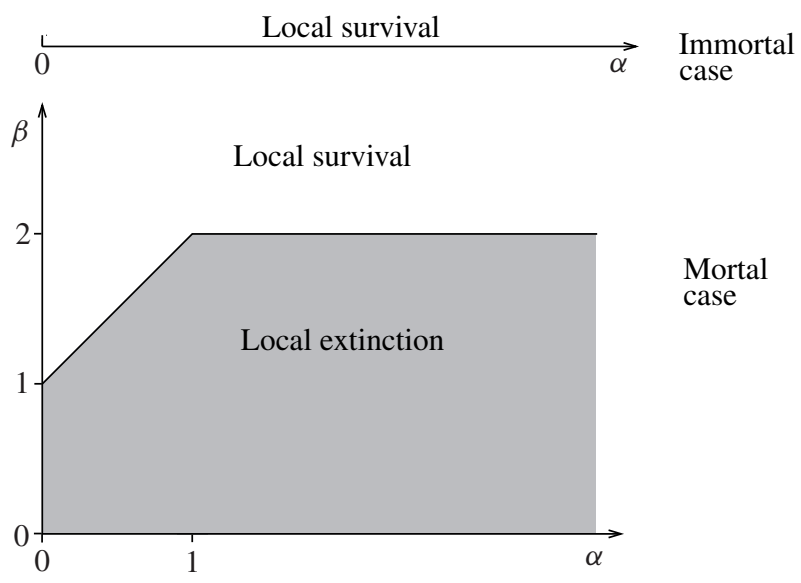

FIgURe 4: The left-drift case.

Corollary 3.1. Let $1-p_{n} \sim 1 / n^{\beta}$ as $n \rightarrow \infty$ (where $\left.\beta>0\right)$.

(a) If $l_{n}-\frac{1}{2} \sim 1 / n^{\alpha}$ as $n \rightarrow \infty$ (where $\alpha>0$ ) there is always global survival; moreover, there is local survival if and only if $\beta \geq \min (2,1+\alpha)$.

(b) If $\frac{1}{2}-l_{n} \sim 1 / n^{\alpha}$ as $n \rightarrow \infty$ (where $\alpha>0$ ) there is always global survival; moreover, there is local survival if and only if $\beta \geq 2$ and $\alpha \geq 1$.

The phase diagram given by Corollary 3.1 can be compared with the results of Examples 2.1 and 2.2; see Figures 3 and 4.

\section{Final remarks}

First of all we note that all our results in the immortal case apply to the inhomogeneous case where there is one particle at all vertices $\left\{n_{k}\right\}_{k \in \mathbb{N}}$ (where $\left\{n_{k}\right\}_{k \in \mathbb{N}}$ is a strictly increasing 
sequence in $\mathbb{N}$ such that $n_{k+1}-n_{k} \leq m<+\infty$ for all $k \in \mathbb{N}$ ) and no particles elsewhere. The generalization is straightforward: all sums and products run over the set of initially occupied vertices $\left\{n_{k}: k \in \mathbb{N}\right\}$ instead of $\mathbb{N}$; in particular, Theorem 2.1 holds in the inhomogeneous case for any generic subset $\left\{n_{k}: k \in \mathbb{N}\right\}$ of occupied vertices (without restrictions). On the other hand the results in the mortal case, since $p_{n}$ can be equal to 0 , deal simultaneously with the homogeneous case as well as with the inhomogeneous case.

Let us discuss briefly the case where there are particles on the whole line $\mathbb{Z}$. When we say that the left-process (respectively right- process) survives globally (respectively locally) we are talking about the process which involves just the particle in the left (respectively right) side of the line (the origin is included). Clearly if either the right-process or the left-process survives (globally or locally) then the whole process survives (globally or locally). We consider the immortal particle case for simplicity and we sketch the differences with the mortal case. Suppose that all the particles in the left-process (respectively right-process) are activated then the probability of local survival is 1 or 0 depending on the divergence or convergence of the series $\sum_{n} \min \left(1,\left(\left(1-l_{n}\right) / l_{n}\right)^{n}\right)\left(\right.$ respectively $\left.\sum_{n} \min \left(1,\left(l_{n} /\left(1-l_{n}\right)\right)^{n}\right)\right)$.

If the probabilities of local survival of both the left and right processes are 0 , then there is local extinction for the whole process as well. Indeed, there might be cooperation between the particles in two half-lines in order to improve the activation process, but nothing can be done for local survival. If either the left-process or the right-process can survive globally, then there is a positive probability of local survival only if at least one of the two processes survives locally (once all the particles are activated). Here we are not saying that one of the processes survives locally by itself but that it survives with positive probability once all its particles are activated (maybe by one particle from the other side). We observe that in the mortal particle case, the local survival of the whole process is equivalent to the local survival of one of the two half-processes by itself.

If both processes cannot survive globally then there might still be global survival; in order to survive globally it is sufficient (and necessary as well) that an infinite number of particles from each side crosses the origin and goes to the other side. In this case, global and local survival are equivalent.

Another question is what can be said in a random environment, that is the case where $\left\{l_{n}\right\}_{n \in \mathbb{N}}$ is a sequence of independent random variables taking values in $(0,1)$ (also the sequence $\left\{p_{n}\right\}_{n \in \mathbb{N}}$ may be randomly chosen). The analysis of the random environment case exceeds the purpose of this paper, nevertheless many results may be deduced from ours. Here is an example in the immortal particle case (with right drift). A realization of the environment is a fixed realization $\left\{l_{n}(\omega)\right\}_{n \in \mathbb{N}}$.

Theorem 4.1. Suppose that $\left\{l_{n}\right\}_{n \in \mathbb{N}}$ is a sequence of independent random variables such that $\mathbb{P}\left(l_{n}<\frac{1}{2}\right)=1$ for all $n \in \mathbb{N}$. Then either the probability of local survival is 1 for almost every realization of the environment or it is 0 for almost every realization of the environment.

In particular, we have the following sufficient conditions.

(a) If $\sum_{n \in \mathbb{N}} \mathbb{P}\left(n\left(\frac{1}{2}-l_{n}\right) \leq M\right)=+\infty$ for some $M$, then the probability of local survival is 1 for almost every realization of the environment.

(b) If

$$
\sum_{n \in \mathbb{N}} \mathbb{P}\left(\frac{n\left(\frac{1}{2}-l_{n}\right)}{\ln (n)}>\frac{1+\ln (\ln (n)) / \ln (n)}{4-2(\ln (n)+\ln (\ln (n))) / n}\right)<+\infty,
$$

then the probability of local survival is 1 for almost every realization of the environment. 
(c) If

$$
\sum_{n \in \mathbb{N}} \mathbb{P}\left(\frac{n\left(\frac{1}{2}-l_{n}\right)}{\ln (n)}<\frac{1+\beta \ln (\ln (n)) / \ln (n)}{4-2(\ln (n)+\beta \ln (\ln (n))) / n}\right)<+\infty
$$

for some $\beta>1$, then the probability of local survival is 0 for almost every realization of the environment.

Theorem 4.1 is a consequence of Theorem 2.1, Corollary 2.1, and the Borel-Cantelli lemmas. We note, in particular, that for conditions (b) and (c) we do not need the independence of $\left\{l_{n}\right\}_{n \in \mathbb{N}}$.

\section{Proofs}

Proof of Theorem 2.1. (a) Let $C_{0}$ be the event that the particle which starts at 0 visits all vertices $n \geq 1$ : since $l_{0}<\frac{1}{2}$, then $\mathbb{P}\left(B_{\infty}\right)=\mathbb{P}\left(C_{0}\right)=1$. Moreover, with respect to $\mathbb{P}\left(\cdot \mid C_{0}\right)$, $\left\{A_{n}\right\}_{n \geq 1}$ is an independent family of events; $\mathbb{P}\left(A_{n}\right)=\mathbb{P}\left(A_{n} \mid B_{n}\right)=\mathbb{P}\left(A_{n} \mid C_{0}\right)$ for $n \geq 1$. Clearly, in this case, $\left\{A_{n}\right\}_{n \geq 1}$ is independent with respect to $\mathbb{P}$. Thus,

$$
\sum_{n \in \mathbb{N}} \mathbb{P}\left(A_{n}\right)=\sum_{n \in \mathbb{N}}\left(\frac{l_{n}}{1-l_{n}}\right)^{n}
$$

The claim follows from the Borel-Cantelli lemmas.

(b) If all particles have a drift to the left, each particle visits 0 almost surely only a finite number of times. Hence, in order to have local survival, we need to activate all particles. But infinite activation is also a sufficient condition since starting at $n>0$ each particle visits 0 almost surely at least once.

Before proving Corollary 2.1 we need a technical lemma.

Lemma 5.1. Let $W=\sum_{i=1}^{\infty}\left(1-a_{n}\right)^{n}$ (where $a_{n} \in[0,1]$ for all $\left.n \geq 1\right)$, then the following conditions hold.

(a) The inequality $\lim \inf _{n} n a_{n}<+\infty$ implies $W=+\infty$.

(b) If, for every sufficiently large $n, a_{n} \leq n^{-1}(\ln (n)+\ln (\ln (n)))$ then $W=+\infty$.

(c) If there exists $\beta>1$ such that, for every sufficiently large $n, a_{n} \geq n^{-1}(\ln (n)+\beta \ln (\ln (n)))$ then $W<+\infty$.

Proof. (a) Let $\left\{n_{j}\right\}_{j \in \mathbb{N}}$ and $M>0$ such that $a_{n_{j}} \leq M / n_{j}$ for all $j \in \mathbb{N}$. Hence, $\left(1-a_{n_{j}}\right)^{n_{j}} \geq$ $\left(1-M / n_{j}\right)^{n_{j}} \rightarrow \mathrm{e}^{-M}$ as $j \rightarrow \infty$.

(b) Since, eventually as $n \rightarrow \infty,\left(1-a_{n}\right)^{n} \geq\left(1-q_{n}\right)^{n}$, where $q_{n}=\ln (n) / n+\ln (\ln (n)) / n$, it is enough to prove that $W^{\prime}:=\sum_{n \geq 1}\left(1-q_{n}\right)^{n}=+\infty$. To this aim, we prove that there exists 
$c>0$ such that $\left(1-q_{n}\right)^{n} \geq c /(n \ln (n))$ for all $n \geq 2$. Indeed, let $f(x):=x \ln (x)(1-$ $\ln (x) / x-\ln (\ln (x)) / x)^{x}$ (for all $x \geq 2$ ), then

$$
\begin{aligned}
f(x)= & \exp \left(x \ln \left(1-\frac{\ln (x)}{x}-\frac{\ln (\ln (x))}{x}\right)+\ln (x)+\ln (\ln (x))\right) \\
= & \exp \left(x \left(-\frac{\ln (x)+\ln (\ln (x))}{x}-\frac{(\ln (x)+\ln (\ln (x)))^{2}}{2 x^{2}}\right.\right. \\
& \left.\left.\quad+O\left(\left|\frac{\ln (x)+\ln (\ln (x))}{x}\right|^{3}\right)\right)+\ln (x)+\ln (\ln (x))\right) \\
= & \exp \left(-\frac{(\ln (x)+\ln (\ln (x)))^{2}}{2 x}\left(1+O\left(\left|\frac{\ln (x)+\ln (\ln (x)) \mid)))}{x}\right|\right)\right.\right. \\
\rightarrow & 1^{-} \quad \text { as } x \rightarrow \infty
\end{aligned}
$$

(where, as usual, given two functions $h$ and $f$, by $h=O(f)$ as $x \rightarrow x_{0}$ we mean $\left.\limsup \operatorname{sux}_{x \rightarrow x_{0}}|h(x) / f(x)|<\infty\right)$. Hence, by continuity and compactness, since $f(x)>0$ for all $x \geq 2$, there exists $c>0$ such that $f(x) \geq c$ for all $x \geq 2$.

(c) Take $\beta>1$ such that $a_{n} \geq n^{-1}(\ln (n)+\beta \ln (\ln (n)))=: q_{n}$ for every sufficiently large $n$, then

$$
\begin{aligned}
\left(1-a_{n}\right)^{n} & \leq\left(1-q_{n}\right)^{n} \\
& \leq\left(\left(1-q_{n}\right)^{1 / q_{n}}\right)^{n q_{n}} \\
& <\exp \left(-n q_{n}\right) \\
& \leq \exp (-\ln (n)-\beta \ln (\ln (n))) \\
& =\frac{1}{n \ln (n)^{\beta}}
\end{aligned}
$$

hence, $W<+\infty$.

The proof of Lemma 5.1 implies, in particular, that if, for every sufficiently large $n, a_{n} \leq$ $(\ln (n)+\ln (\ln (n))) / n$ then $\sum_{i \in J}^{\infty}\left(1-a_{n}\right)^{n}=+\infty$ for every $J \subseteq \mathbb{N} \backslash\{0\}$ such that $\sum_{i \in J}^{\infty} 1 /(n \ln (n))=+\infty$.

Proof of Corollary 2.1. Rewrite $l_{n} /\left(1-l_{n}\right)$ as $1-a_{n}$, where $a_{n}:=\left(1-2 l_{n}\right) /\left(1-l_{n}\right)$.

(a) We note that $\lim _{n f} n\left(\frac{1}{2}-l_{n}\right)<\infty$ is equivalent to $\lim \inf _{n} n a_{n}<\infty$; thus, according to Lemma 5.1(a), $\sum_{n=1}^{\infty}\left(l_{n} /\left(1-l_{n}\right)\right)^{n}$ diverges. Theorem 2.1 yields the result.

(b) Since $n\left(\frac{1}{2}-l_{n}\right) / \ln (n) \leq(1+\ln (\ln (n)) / \ln (n)) /(4-2(\ln (n)+\ln (\ln (n))) / n)$ is equivalent to $a_{n} \leq \ln (n) / n+\ln (\ln (n)) / n$, then, according to Lemma $5.1(\mathrm{~b}), \sum_{n=1}^{\infty}\left(l_{n} /\left(1-l_{n}\right)\right)^{n}$ diverges and Theorem 2.1 yields the result.

(c) We note that the inequality $n\left(\frac{1}{2}-l_{n}\right) / \ln (n) \geq(1+\beta \ln (\ln (n)) / \ln (n)) /(4-2(\ln (n)+$ $\beta \ln (\ln (n))) / n)$ is equivalent to $a_{n} \geq \ln (n) / n+\beta \ln (\ln (n)) / n$; thus, according to Lemma 5.1(c), the series $\sum_{n=1}^{\infty}\left(l_{n} /\left(1-l_{n}\right)\right)^{n}$ converges and Theorem 2.1 yields the result.

(d) Note that $\left(1-\left(1-2 l_{n}\right) /\left(1-l_{n}\right)\right)^{\left(1-l_{n}\right) /\left(1-2 l_{n}\right)}<1 /$ e. Then

$$
\left(\frac{l_{n}}{1-l_{n}}\right)^{n}<\exp \left(-\frac{2 n\left(\frac{1}{2}-l_{n}\right)}{1-l_{n}}\right) .
$$


We divide the sum into two series as follows:

$$
\sum_{n}\left(\frac{l_{n}}{1-l_{n}}\right)^{n}<\sum_{n: 2 /\left(1-l_{n}\right) \leq \lambda}\left(\frac{l_{n}}{1-l_{n}}\right)^{n}+\sum_{n: 2 /\left(1-l_{n}\right)>\lambda} \exp \left(-\frac{2 n\left(\frac{1}{2}-l_{n}\right)}{1-l_{n}}\right) .
$$

The second series is finite since each summand is bounded from above by $\exp \left(-\lambda n\left(\frac{1}{2}-l_{n}\right)\right)$. The first series is finite since $2 /\left(1-l_{n}\right) \leq \lambda$ implies $l_{n} /\left(1-l_{n}\right) \leq \lambda / 2-1<1$. Again, Theorem 2.1 yields the conclusion.

Proof of Proposition 2.1. The proof is based on a comparison between our frog model and the heterogeneous firework process introduced in [9]. Consider the explicit construction of the frog model given in Section 1. Define a family of independent random variables $\left\{R_{i}\right\}_{i \in \mathbb{N}}$ as $R_{i}:=\max \left\{Z_{n}^{i}: n \in \mathbb{N}\right\}$, that is, the maximum right-excursion of the independent walker $\left\{Z_{n}^{i}\right\}_{n \in \mathbb{N}}$. Conditioned on the activation of the $i$ th walker, that is $T_{i}<\infty, R_{i}$ is also the maximum right-excursion of the dependent walker $\left\{X_{n}^{i}\right\}_{n \in \mathbb{N}}$ of our frog model. Let $\left\{R_{i}\right\}_{i \in \mathbb{N}}$ be the radii of the firework process. In the firework process sites are activated as follows: at time 0 , site 0 sends a signal and activates all the sites to its right up to a distance $R_{0}$. Iteratively, if site $i$ is activated, it sends a signal which will activate all the inactive sites, if any, in the interval $\left(i, i+R_{i}\right]$. We prove that site $i$ is activated in the firework process if and only if the $i$ th walker is activated in our frog model. When $i=0$ there is nothing to prove. Suppose that it holds for all $i \leq N$. The site $N+1$ is activated if and only if all the sites between 0 and $N$ are activated and $N+1 \leq R_{i}+i$ for some $i \leq N$. By induction, this is equivalent to the event 'all the walkers starting between 0 and $N$ are activated and, for some $i \leq N$, the $i$ th walker reaches $N+1$ ', that is, the $(N+1)$ th walker is activated.

Following the proof of [9, Proposition 3.3] (in that paper $B_{n}$ is denoted by $V_{n}$ ), we note that

$$
B_{n_{k+1}} \supseteq B_{n_{k}} \cap\left(\bigcup_{i=0}^{n_{k}}\left(R_{i} \geq n_{k+1}-i\right)\right)
$$

By basic random walk theory, $\mathbb{P}\left(R_{i} \geq k\right)=\left(1-l_{i}\right)^{k} / l_{i}^{k}$. Using the Fortuin-Kasteleyn-Ginibre inequality and the independence of the $\left\{R_{i}\right\}_{i \in \mathbb{N}}$, we have that

$$
\mathbb{P}\left(B_{n_{k+1}}\right) \geq \mathbb{P}\left(B_{n_{k}}\right)\left(1-\prod_{i=0}^{n_{k}}\left(1-\left(\frac{1-l_{i}}{l_{i}}\right)^{n_{k+1}-i}\right)\right)
$$

hence, the probability of survival of the firework process (and of our frog model as well) is bounded from below by

$$
\prod_{k=0}^{\infty}\left(1-\prod_{i=0}^{n_{k}}\left(1-\left(\frac{1-l_{i}}{l_{i}}\right)^{n_{k+1}-i}\right)\right)
$$

According to Lemma 5.2(b), below, the previous product is strictly positive if and only if

$$
\sum_{k=0}^{\infty} \prod_{i=0}^{n_{k}}\left(1-\left(\frac{1-l_{i}}{l_{i}}\right)^{n_{k+1}-i}\right)<+\infty
$$

Proof of Theorem 2.2. The proof can be easily adapted from the proof of Theorem 3.3. 
Proof of Proposition 2.2. Note that, since $l_{n}>\frac{1}{2}$ for all $n \in \mathbb{N}$, then $\liminf _{n} l_{n}>\frac{1}{2}$ is equivalent to $\inf _{n} l_{n}>1 / 2$. We associate with the process a random walk on a subset of $\mathbb{N} \times \mathbb{N}$. To this aim, we define the generation 0 as the set containing only the initial active particle and, recursively, the generation $n+1$ as the set of vertices visited by at least one particle of generation $n$. We denote by $j_{n+1}$ the right-most position reached by a particle of a generation $i \leq n$. Hence, the generation $n$ is nonempty if and only if $j_{n}>j_{n-1}$, in this case it contains all the particles starting in the set of positions $\left\{j_{n-1}+1, \ldots, j_{n}\right\}$. It is clear that if the $n$th generation is empty then all generations $m \geq n$ are empty as well. The system survives locally if and only if all the particles are activated, that is, if and only if every generation contains at least one particle.

As a warm-up we start with the simpler case of an homogeneous system: $l_{n}=l>\frac{1}{2}$ for every $n$. We associate with this process the random walk $\left\{\Delta_{n}\right\}_{n}$ which counts the particles of the generation $n$, which is $\Delta_{n}=j_{n}-j_{n-1}$. The origin is the only absorbing state of this Markov chain. It is easy to compute the probability of absorption (or local extinction),

$$
\begin{aligned}
\mathbb{P}\left(\Delta_{n}=0 \mid \Delta_{n-1}=h\right) & =\left(1-\frac{1-l}{l}\right)\left(1-\left(\frac{1-l}{l}\right)^{2}\right) \cdots\left(1-\left(\frac{1-l}{l}\right)^{h}\right) \\
& \geq \prod_{i=1}^{\infty}\left(1-\left(\frac{1-l}{l}\right)^{i}\right),
\end{aligned}
$$

which is strictly positive according to Lemma 5.2, below. This implies, in particular, that the Markov chain $\left\{\Delta_{n}\right\}_{n}$ is absorbed in 0 almost surely; whence, $\mathbb{P}\left(B_{\infty}\right)=0$.

In the general case of an inhomogeneous system, $\left\{\Delta_{n}\right\}_{n}$ is no longer a Markov process. In order to be able to mimic the steps above, we must consider the Markov chain $\left\{\left(\Delta_{n}, j_{n}\right)\right\}_{n}$. In this case,

$$
\begin{aligned}
\mathbb{P}\left(\Delta_{n}=0 \mid\left(\Delta_{n-1}, j_{n-1}\right)=(h, k)\right) & =\prod_{i=k-h+1}^{k}\left(1-\left(\frac{1-l_{i}}{l_{i}}\right)^{k-i+1}\right) \\
& \geq \inf _{h, k \in \mathbb{N}: h \leq k} \prod_{i=k-h+1}^{k}\left(1-\left(\frac{1-l_{i}}{l_{i}}\right)^{k-i+1}\right) \\
& =\inf _{k \in \mathbb{N}} \prod_{i=1}^{k}\left(1-\left(\frac{1-l_{i}}{l_{i}}\right)^{k-i+1}\right) .
\end{aligned}
$$

Note that $\inf _{k \in \mathbb{N}} \prod_{i=1}^{k}\left(1-\left(\left(1-l_{i}\right) / l_{i}\right)^{k-i+1}\right)>0$ is equivalent to $\inf _{i \in \mathbb{N}} l_{i}>\frac{1}{2}$ and implies $\mathbb{P}\left(B_{\infty}\right)=0$. In order to check the equivalence, we observe that the ' while the converse comes from the inequality $\prod_{i=1}^{k}\left(1-\left(\left(1-l_{i}\right) / l_{i}\right)^{k-i+1}\right) \leq 1-\left(1-l_{k}\right) / l_{k}$

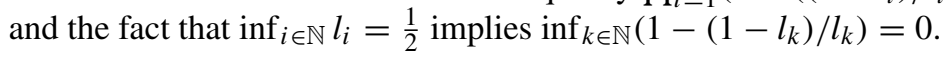

Proof of Proposition 3.1. Suppose that $\sup _{n} p_{n}=p<1$ and that $n$ dormant particles are activated in $n$ consecutive vertices, say $i, i+1, \ldots, i+n-1$. The probability that the lifespan of all these particles is so short that neither of them can possibly reach the vertex $i+n$ (and activate more particles) is

$$
\prod_{j=0}^{n-1}\left(1-p_{i+j}^{n-j}\right) \geq \prod_{j=1}^{\infty}\left(1-p^{j}\right)>0, \quad \text { for all } n \in \mathbb{N} .
$$


As in the proof of Proposition 2.2, $\mathbb{P}\left(\Delta_{n}=0 \mid\left(\Delta_{n-1}, j_{n-1}\right)=(n, i+n-1)\right) \geq \prod_{j=1}^{\infty}(1-$ $\left.p^{j}\right)>0$, and the conclusion follows.

Proof of Theorem 3.1. Define $L_{1}:=\sup _{n \in \mathbb{N}}\left(\max \left(\mathcal{B}_{n+1}\right)-\min \left(\mathcal{B}_{n}\right)\right)$. We note that the series $\sum_{n \in \mathbb{N}} \sum_{k \in \mathcal{B}_{n}}\left(1-p_{k}\right)^{L / 2}$ is convergent; hence, we have that in all but a finite number (say $N_{0}$ ) of blocks at least one particle has a strictly positive lifetime parameter $p_{k}$. Since there is always a positive probability that the particle at 0 reaches $\max \left(\mathscr{B}_{N_{0}+1}\right)$, then we can assume without loss of generality that in every block $\mathscr{B}_{n}$ there is at least one particle with strictly positive lifetime parameter.

Consider the (mortal) random walk with $p(j, j-1)=p_{n} l_{n}, p(j, j+1)=p_{n}\left(1-l_{n}\right)$, and $p(j, D)=1-p_{n}$, for all $j \in \mathbb{Z}$ and $p(D, D)=1$ ( $D$ represents the absorbing state where the particle is considered dead). Define

$$
\begin{gathered}
f_{n}^{(k)}(x, y)=\mathbb{P}(\text { the } n \text {th random walk visits } y \text { for the first time at time } k+h \\
\mid \text { the random walk is at } x \text { at time } h) .
\end{gathered}
$$

Let $F_{n}(x, y \mid z)=\sum_{k} f_{n}^{(k)}(x, y) z^{k}$. Then we obtain

$$
F_{n}(x-1, x \mid z)=p_{n}\left(1-l_{n}\right) z+p_{n} l_{n} z F_{n}(x-1, x+1 \mid z) .
$$

Noting that $F_{n}(x-1, x+1 \mid z)=\left(F_{n}(x-1, x \mid z)\right)^{2}$, we get

$$
F_{n}(x-1, x \mid z)=\frac{1-\sqrt{1-4 z^{2} p_{n}^{2} l_{n}\left(1-l_{n}\right)}}{2 z p_{n} l_{n}}=\frac{2 z p_{n}\left(1-l_{n}\right)}{1+\sqrt{1-4 z^{2} p_{n}^{2} l_{n}\left(1-l_{n}\right)}}
$$

Note also that the probability for a mortal particle starting from $j_{n}$ to ever reach $j_{n+1}$ is $F_{j_{n}}(x-$ $1, x \mid 1)^{j_{n+1}-j_{n}}$.

The probability that, in each block $\mathscr{B}_{n}$ such that $\max \left(\mathscr{B}_{n+1}\right)>\max \left(\mathscr{B}_{n}\right)$, there exists at least one particle which visits all the sites of the following block is bounded from below by the probability that in every block at least one particle has a right-excursion larger than $L_{1}$, that is

$$
\prod_{n=1}^{\infty}\left(1-\prod_{k \in \mathcal{B}_{n}}\left(1-\left(\frac{1-\sqrt{1-4 p_{k}^{2} l_{k}\left(1-l_{k}\right)}}{2 p_{k} l_{k}}\right)^{L_{1}}\right)\right) .
$$

By Lemma 5.2 a sufficient condition for the positivity of the product in (5.2) is

$$
\sum_{n \in \mathbb{N}} \prod_{k \in \mathscr{B}_{n}}\left(1-\left(\frac{1-\sqrt{1-4 p_{k}^{2} l_{k}\left(1-l_{k}\right)}}{2 p_{k} l_{k}}\right)^{L_{1}}\right)<+\infty
$$

the fact that in each block there is at least one particle, say at $k$, with $p_{k}>0$ implies that each term in the infinite product (5.2) is strictly positive and Lemma 5.2 applies. Since $1-x^{n} \leq n(1-x)$ 
(for all $n \in \mathbb{N}$ ) and by using the following estimates:

$$
\begin{aligned}
0 & \leq 1-\frac{2 p_{k}\left(1-l_{k}\right)}{1+\sqrt{1-4 p_{k}^{2} l_{k}\left(1-l_{k}\right)}} \\
& =\frac{1+\sqrt{\left(2 p_{k} l_{k}-1\right)^{2}+4 p_{k}\left(1-p_{k}\right) l_{k}}-2 p_{k}\left(1-l_{k}\right)}{1+\sqrt{1-4 p_{k}^{2} l_{k}\left(1-l_{k}\right)}} \\
& \leq 1-2 p_{k}\left(1-l_{k}\right)+2 \sqrt{1-p_{k}}+\left|2 p_{k} l_{k}-1\right| \\
& \leq W_{k} \quad \text { if } p_{k} l_{k} \leq \frac{1}{2}, \\
& := \begin{cases}2\left(1-p_{k}\right)+2 \sqrt{1-p_{k}} \\
4 p_{k}\left(l_{k}-\frac{1}{2}\right)+2 \sqrt{1-p_{k}} & \text { if } p_{k} l_{k}>\frac{1}{2},\end{cases}
\end{aligned}
$$

we have (5.3) implied by $\sum_{n \in \mathbb{N}} \prod_{k \in B_{n}} W_{k}<+\infty$ which, in turn, is implied by

where

$$
\sum_{n \in \mathbb{N}} \prod_{k \in B_{n}} S_{k}<+\infty
$$

$$
S_{k}:= \begin{cases}\sqrt{1-p_{k}} & \text { if } p_{k} l_{k} \leq \frac{1}{2} \\ \sqrt{1-p_{k}}+2 p_{k}\left(l_{k}-\frac{1}{2}\right) & \text { if } p_{k} l_{k}>\frac{1}{2}\end{cases}
$$

since $\prod_{k \in B_{n}} W_{k} \leq 4^{L} \prod_{k \in B_{n}} S_{k}$.

By using the inequality between arithmetic and geometric means, we have

$$
\sum_{n \in \mathbb{N}} \prod_{k \in \mathscr{B}_{n}} S_{k} \leq \frac{1}{L} \sum_{n \in \mathbb{N}} \sum_{k \in \mathscr{B}_{n}} S_{k}^{L}
$$

Hence, $\sum_{n \in \mathbb{N}} \sum_{k \in B_{n}} S_{k}^{L}<+\infty$ implies global survival. However, using, on the one hand, the Minkowski inequality and, on the other hand, the fact that $S_{k}$ is the sum of the two nonnegative functions $\sqrt{1-p_{k}}$ and $2 p_{k}\left(l_{k}-\frac{1}{2}\right) \mathbf{1}_{(0,+\infty)}\left(p_{k} l_{k}-\frac{1}{2}\right)$, we have that $\sum_{n \in \mathbb{N}} \sum_{k \in B_{n}} S_{k}^{L}<+\infty$ is equivalent to $\sum_{n \in \mathbb{N}} \sum_{k \in B_{n}}\left(1-p_{k}\right)^{L / 2}<+\infty$ and

$$
\sum_{n=0}^{\infty} \sum_{k \in \mathcal{B}_{n}: p_{k} l_{k}>1 / 2}\left(l_{k}-\frac{1}{2}\right)^{L}<+\infty
$$

(since, in both cases, $p_{k} \rightarrow 1$ as $k \rightarrow \infty, k \in \bigcup_{n \in \mathbb{N}} \mathscr{B}_{n}$ ).

Proof of Theorem 3.2. We note that, in this case, by switching $l_{n}$ and $1-l_{n}$ in (5.1), we have

$$
\mathbb{P}\left(A_{n} \mid B_{n}\right)=\left(\frac{1-\sqrt{1-4 p_{n}^{2} l_{n}\left(1-l_{n}\right)}}{2 p_{n}\left(1-l_{n}\right)}\right)^{n}=\left(\frac{2 p_{n} l_{n}}{1+\sqrt{1-4 p_{n}^{2} l_{n}\left(1-l_{n}\right)}}\right)^{n} .
$$

Now, since $A_{n} \subset B_{n}, \mathbb{P}\left(A_{n}\right) \leq \mathbb{P}\left(A_{n} \mid B_{n}\right)$ and by the Borel-Cantelli lemma, we have that $\sum_{n} \mathbb{P}\left(A_{n} \mid B_{n}\right)<+\infty$ implies $\mathbb{P}\left(A_{i}\right.$ i.o. $)=0$.

(a) Note that it is enough to prove the result in the case $\Delta_{n} \rightarrow 0$ and $\delta_{n} \rightarrow 0$. The result in the general case follows from a coupling between the process and a similar one with $p_{n}^{\prime}=1-\Delta_{n}^{\prime}$ and $l_{n}^{\prime}=1-\delta_{n}^{\prime}$ such that $\Delta_{n} \geq \Delta_{n}^{\prime} \rightarrow 0, \delta_{n}^{\prime}=\delta_{n}$ if $\delta_{n}<0,0 \leq \delta_{n}^{\prime} \leq \delta_{n}$ if $\delta_{n} \geq 0, \delta_{n}^{\prime} \rightarrow 0$, 
and $\liminf _{n \rightarrow \infty} n\left(2 \delta_{n}^{\prime}+\sqrt{2 \Delta_{n}^{\prime}+4{\delta_{n}^{\prime}}^{2}}\right) / \ln (n)>1$. Hence, from now on we suppose that $\Delta_{n} \rightarrow 0$ and $\delta_{n} \rightarrow 0$ as $n \rightarrow \infty$.

In order to check whether the series $\sum_{n} \mathbb{P}\left(A_{n} \mid B_{n}\right)$ is convergent, we use Lemma 5.1; hence, it is important to estimate $a_{n}=1-\left(1-\sqrt{1-4 p_{n}^{2} l_{n}\left(1-l_{n}\right)}\right) /\left(2 p_{n}\left(1-l_{n}\right)\right)$. To this aim, we write

$$
\begin{aligned}
\mathbb{P}\left(A_{n} \mid B_{n}\right)^{1 / n} & =\frac{\left(1-\Delta_{n}\right)\left(1-2 \delta_{n}\right)}{1+\sqrt{1-\left(1-\Delta_{n}\right)^{2}\left(1-4 \delta_{n}^{2}\right)}} \\
& =\left(1-\Delta_{n}\right)\left(1-2 \delta_{n}\right)\left(1-\sum_{j=1}^{\infty}(-1)^{j+1}\left(1-\left(1-\Delta_{n}\right)^{2}\left(1-4 \delta_{n}^{2}\right)\right)^{j / 2}\right) \\
& =\left(1-\Delta_{n}\right)\left(1-2 \delta_{n}\right)\left(1-\sqrt{2 \Delta_{n}+4 \delta_{n}^{2}}+o\left(\sqrt{\Delta_{n}+2 \delta_{n}^{2}}\right)\right) \\
& =1-\left(2 \delta_{n}+\sqrt{2 \Delta_{n}+4 \delta_{n}^{2}}\right)+o\left(\sqrt{2 \Delta_{n}+4 \delta_{n}^{2}}\right)
\end{aligned}
$$

as $\Delta_{n} \rightarrow 0$ and $\delta_{n} \rightarrow 0$ (where, as usual, given two functions $h$ and $f$, by $h=o(f)$ as $x \rightarrow x_{0}$ we mean that $\left.\lim _{x \rightarrow x_{0}} h(x) / f(x)=0\right)$. If $\liminf _{n \rightarrow \infty} n\left(2 \delta_{n}+\sqrt{2 \Delta_{n}+4 \delta_{n}^{2}}\right) / \ln (n)>1$, then $\liminf \inf _{n \rightarrow \infty} n\left(2 \delta_{n}+\sqrt{2 \Delta_{n}+4 \delta_{n}^{2}}+o\left(\sqrt{2 \Delta_{n}+4 \delta_{n}^{2}}\right)\right) / \ln (n)>1$. Thus, according to Lemma 5.1(c), the series $\sum_{n} \mathbb{P}\left(A_{n} \mid B_{n}\right)$ converges.

(b) Given the following estimate:

$$
\mathbb{P}\left(A_{n} \mid B_{n}\right)^{1 / n}=\frac{2 p_{n} l_{n}}{1+\sqrt{1-4 p_{n}^{2} l_{n}\left(1-l_{n}\right)}} \leq \min \left(p_{n}, 2 p_{n} l_{n}\right)=p_{n}\left(1-\left(1-2 l_{n}\right)^{+}\right),
$$

we have that $\sum_{n} p_{n}^{n}\left(1-\left(1-2 l_{n}\right)^{+}\right)^{n}<+\infty$ implies $\sum_{n} \mathbb{P}\left(A_{n} \mid B_{n}\right)<+\infty$.

(c) This follows from $\mathbb{P}\left(A_{n} \mid B_{n}\right)^{1 / n} \leq p_{n}$ for all $n \in \mathbb{N}$.

Proof of Theorem 3.3. Consider the following conditions:

$$
\left\{\begin{array}{c}
\sum_{k \in \cup_{n \in \mathbb{N}}}\left(l_{k}-\frac{1}{2}\right)^{L}+\sum_{k \in p_{k} l_{k}>1 / 2}\left(1-p_{k}\right)^{L / 2}<+\infty, \\
\sum_{k \in \mathcal{O}}\left(\frac{1-\sqrt{1-4 p_{k}^{2} l_{k}\left(1-l_{k}\right)}}{2 p_{k}\left(1-l_{k}\right)}\right)^{k}=+\infty .
\end{array}\right.
$$

The first line of (5.5), according to Theorem 3.1, implies global survival and the activation of every particle. The divergence of the series in the second line of (5.5) implies that, once activated, an infinite number of particles visits the origin (whence we have local survival).

We are left to prove that each of the three conditions implies that the series in the second line of (5.5) is divergent.

(a) Given a sequence $\left\{k_{j}\right\}_{j \in \mathbb{N}}$ in $\mathcal{O}$ such that $\lim _{j \rightarrow \infty} k_{j}\left(2 \delta_{k_{j}}+\sqrt{2 \Delta_{k_{j}}+4 \delta_{k_{j}}^{2}}\right)<\infty$, then $\Delta_{k_{j}} \rightarrow 0$ and $\delta_{k_{j}} \rightarrow 0$ as $j \rightarrow \infty$. Elementary but tedious computations show that, the asymptotic estimate of $a_{k_{j}}=1-\left(1-\sqrt{1-4 p_{k_{j}}^{2} l_{k_{j}}\left(1-l_{k_{j}}\right)}\right) /\left(2 p_{k_{j}}\left(1-l_{k_{j}}\right)\right)$ given by (5.4), can be refined obtaining

$$
\frac{1-\sqrt{1-4 p_{k_{j}}^{2} l_{k_{j}}\left(1-l_{k_{j}}\right)}}{2 p_{k_{j}}\left(1-l_{k_{j}}\right)} \geq 1-\left(2 \delta_{k_{j}}+\sqrt{2 \Delta_{k_{j}}+4 \delta_{k_{j}}^{2}}\right) .
$$


Thus, $\lim _{j \rightarrow \infty} k_{j} a_{k_{j}}<\infty$; hence, according to Lemma 5.1(a), the series in the second line of (5.5) is divergent.

(b) In this case $\Delta_{k} \rightarrow 0$ and $\delta_{k} \rightarrow 0$ as $k \rightarrow \infty, k \in \mathcal{O}$. The conclusion follows from Lemma 5.1(b) as before, since $a_{k} \leq 2 \delta_{k}+\sqrt{2 \Delta_{k}+4 \delta_{k}^{2}} \leq \ln (k) / k$, for all sufficiently large $k \in \mathcal{O}$. To be precise, since the sum in the second line of (5.5) spans in $\mathcal{O}$ we cannot apply Lemma 5.1(b) as it is. Nevertheless, the same argument as in the proof of Lemma 5.1(b) holds in this case as well; in particular, under our hypotheses, the $k$ th summand of the series in the second line of (5.5) is bounded from below by $c / k$ for some $c>0$ and, $\operatorname{since} \sup _{n \in \mathbb{N}}\left(\max \left(\mathscr{B}_{2 n+2}\right)-\right.$ $\left.\min \left(\mathscr{B}_{2 n}\right)\right)<+\infty, \sum_{k \in \mathcal{O}} 1 / k=+\infty$ (see the discussion after the proof of Lemma 5.1).

(c) As in the proof of Theorem 3.1 we assume, without loss of generality, that in every block $\mathcal{B}_{n}$ there is at least one particle with strictly positive lifetime parameter.

If we prove that

$$
\sum_{n \in \mathbb{N}} \prod_{k \in B_{2 n+1}}\left(1-\left(\frac{1-\sqrt{1-4 p_{k}^{2} l_{k}\left(1-l_{k}\right)}}{2 p_{k}\left(1-l_{k}\right)}\right)^{k}\right)<+\infty
$$

then we have that

$$
\prod_{k \in \mathcal{O}}\left(1-\left(\frac{1-\sqrt{1-4 p_{k}^{2} l_{k}\left(1-l_{k}\right)}}{2 p_{k}\left(1-l_{k}\right)}\right)^{k}\right)=0,
$$

which, by Lemma 5.2, below, is equivalent to the second line of (5.5).

Since $(1-x)^{k} \leq k(1-x)$, by using the following estimate:

$$
\begin{aligned}
0 & \leq 1-\frac{2 p_{n} l_{n}}{1+\sqrt{1-4 p_{n}^{2} l_{n}\left(1-l_{n}\right)}} \\
& \leq \widetilde{W}_{n} \\
& := \begin{cases}2\left(1-p_{n}\right)+2 \sqrt{1-p_{n}} & \text { if } p_{n}\left(1-l_{n}\right) \leq \frac{1}{2}, \\
4 p_{n}\left(\frac{1}{2}-l_{n}\right)+2 \sqrt{1-p_{n}} & \text { if } p_{n}\left(1-l_{n}\right)>\frac{1}{2},\end{cases}
\end{aligned}
$$

we have that (5.6) is implied by $\sum_{n \in \mathbb{N}} \prod_{k \in B_{2 n+1}} k \widetilde{W}_{k}<+\infty$ which, in turn, is implied by $\sum_{n \in \mathbb{N}} \prod_{k \in B_{2 n+1}} k \widetilde{S}_{k}<+\infty$, where

$$
\widetilde{S}_{n}:= \begin{cases}\sqrt{1-p_{n}} & \text { if } p_{n}\left(1-l_{n}\right) \leq \frac{1}{2}, \\ 2 p_{n}\left(\frac{1}{2}-l_{n}\right)+\sqrt{1-p_{n}} & \text { if } p_{n}\left(1-l_{n}\right)>\frac{1}{2}\end{cases}
$$

since $\prod_{k \in \mathscr{B}_{2 n+1}} k \widetilde{W}_{k} \leq 4^{L} \prod_{k \in B_{2 n+1}} k \widetilde{S}_{k}$. The conclusion follows using the inequality between arithmetic and geometric means and the Minkowski inequality as in the proof of Theorem 3.1.

Proof of Corollary 3.1. If $L>\max (2 / \beta, 1 / \alpha)$ then $\sum_{n \in \mathbb{N}}\left(\Delta^{L / 2}+\left|\delta_{n}\right|^{L}\right)<\infty$; hence, according to Theorem 3.1, for all $\alpha, \beta>0$ there is global survival in both cases.

Define $r_{n}:=2 \delta_{n}+\sqrt{2 \Delta_{n}+4 \delta_{n}^{2}} \equiv 2 \Delta_{n} /\left(\sqrt{2 \Delta_{n}+4 \delta_{n}^{2}}-2 \delta_{n}\right)$, where, using the same notation as in Theorems 3.2 and 3.3, $p_{n}=1-\Delta_{n}$ and $l_{n}=\frac{1}{2}-\delta_{n}$. 
(a) Suppose that $\Delta_{n} \sim 1 / n^{\beta}$ and $\delta_{n} \sim-1 / n^{\alpha}$. Elementary computations show that, as $n \rightarrow \infty$,

$$
r_{n} \sim \begin{cases}C_{\alpha, \beta} \sqrt{\Delta_{n}} \sim \frac{C_{\alpha, \beta}}{n^{\beta / 2}} & \text { if } \beta \leq 2 \alpha, \\ C_{\alpha, \beta} \frac{\Delta_{n}}{\delta_{n}} \sim \frac{C_{\alpha, \beta}}{n^{\beta-\alpha}} & \text { if } \beta>2 \alpha,\end{cases}
$$

where

$$
C_{\alpha, \beta}= \begin{cases}\sqrt{2} & \text { if } \beta<2 \alpha \\ \sqrt{6}-2 & \text { if } \beta=2 \alpha \\ \frac{1}{2} & \text { if } \beta>2 \alpha\end{cases}
$$

thus,

$$
\lim _{n \rightarrow \infty} \frac{n r_{n}}{\ln (n)}= \begin{cases}0 & \text { if }\{\beta \leq 2 \alpha, \beta \geq 2\} \cup\{\beta>2 \alpha, \beta \geq 1+\alpha\} \equiv\{\beta \geq \min (2,1+\alpha)\} \\ +\infty & \text { if }\{\beta \leq 2 \alpha, \beta<2\} \cup\{\beta>2 \alpha, \beta<1+\alpha\} \equiv\{\beta<\min (2,1+\alpha)\}\end{cases}
$$

(b) Suppose that $\Delta_{n} \sim 1 / n^{\beta}$ and $\delta_{n} \sim 1 / n^{\alpha}$. Since $\max \left(4 \delta_{n}, \sqrt{2 \Delta_{n}}\right) \leq r_{n} \leq 4 \delta_{n}+\sqrt{2 \Delta_{n}}$ we have that, for every $\alpha, \beta>0$, there exists $\varepsilon \in(0,1)$ such that

$$
(1-\varepsilon) \frac{n}{\ln (n)} \max \left(\frac{4}{n^{\alpha}}, \sqrt{\frac{2}{n^{\beta}}}\right) \leq \frac{n r_{n}}{\ln (n)} \leq(1+\varepsilon) \frac{n}{\ln (n)}\left(\frac{4}{n^{\alpha}}+\sqrt{\frac{2}{n^{\beta}}}\right),
$$

for every sufficiently large $n \in \mathbb{N}$. Whence,

$$
\lim _{n \rightarrow \infty} \frac{n r_{n}}{\ln (n)}= \begin{cases}0 & \text { if }\{\beta \geq 2, \alpha \geq 1\} \\ +\infty & \text { if }\{\beta<2\} \cup\{\alpha<1\}\end{cases}
$$

The conclusion follows as before.

Lemma 5.2. Let $\left\{\alpha_{i}\right\}_{i \in \mathbb{N}}$ and $\left\{k_{i}\right\}_{i \in \mathbb{N}}$ be such that $\alpha_{i} \in(-\infty, 1)$ and $k_{i} \geq 0$ for all $i \in \mathbb{N}$.

(a) We have

$$
\sum_{i \in \mathbb{N}} k_{i} \alpha_{i}<+\infty \Longleftarrow \prod_{i \in \mathbb{N}}\left(1-\alpha_{i}\right)^{k_{i}}>0 .
$$

(b) Moreover, if $\alpha_{i} \in[0,1)$ and $k_{i} \geq 1$ eventually as $i \rightarrow \infty$, then

$$
\sum_{i \in \mathbb{N}} k_{i} \alpha_{i}<+\infty \quad \Longleftrightarrow \quad \prod_{i \in \mathbb{N}}\left(1-\alpha_{i}\right)^{k_{i}}>0
$$

(c) If $\alpha_{i}(j) \in[0,1-\epsilon]$ (for some $\epsilon>0$ ) and $k_{i}(j) \geq 1$ for all $i, j \in \mathbb{N}$, then

$$
\sup _{j \in \mathbb{N}} \sum_{i \in \mathbb{N}} k_{i}(j) \alpha_{i}(j)<+\infty \quad \Longleftrightarrow \quad \inf _{j \in \mathbb{N}} \prod_{i \in \mathbb{N}}\left(1-\alpha_{i}(j)\right)^{k_{i}(j)}>0 .
$$

Proof. Clearly $\prod_{i \in \mathbb{N}}\left(1-\alpha_{i}\right)^{k_{i}}>0$ if and only if $\sum_{i \in \mathbb{N}} k_{i} \ln \left(1-\alpha_{i}\right)>-\infty$.

(a) Observe that $\ln (1-x) \leq-x$ for all $x<1$; hence,

$$
\sum_{i \in \mathbb{N}} k_{i} \alpha_{i} \leq-\sum_{i \in \mathbb{N}} k_{i} \ln \left(1-\alpha_{i}\right)<\infty
$$


(b) In this case, since $k_{i} \geq 1$ both sides imply $\alpha_{i} \rightarrow 0$. Thus, $\ln \left(1-\alpha_{i}\right) \sim-\alpha_{i}$ and

$$
\sum_{i \in \mathbb{N}} k_{i} \ln \left(1-\alpha_{i}\right)>-\infty \quad \Longleftrightarrow \quad \sum_{i \in \mathbb{N}} k_{i} \alpha_{i}<\infty .
$$

(c) If $\inf _{j \in \mathbb{N}} \prod_{i \in \mathbb{N}}\left(1-\alpha_{i}(j)\right)^{k_{i}(j)}>0$ then using the first inequality in (5.7) we obtain $\sup _{j \in \mathbb{N}} \sum_{i \in \mathbb{N}} k_{i}(j) \alpha_{i}(j)<+\infty$. Conversely, it suffices to note that there exists $\delta \in(0,1)$ such that $-\delta \alpha_{i}(j) \leq \ln \left(1-\alpha_{i}(j)\right)$.

Lemma 5.3. Let $\left\{\alpha_{i}\right\}_{i}$ be a sequence of nonnegative numbers. Define $\bar{\alpha}_{n}:=\min \left\{\alpha_{i}: i \leq n\right\}$. The following two statements are equivalent.

(a) There exists an increasing sequence $\left\{n_{i}\right\}$ such that $\sum_{i}\left(n_{i+1}-n_{i}\right) \alpha_{n_{i}}<+\infty$.

(b) Either $\alpha_{n}=0$ for infinitely many $n \in \mathbb{N}$ or it is possible to define recursively an infinite, increasing sequence $\left\{r_{j}\right\}_{j}$ by

$$
\left\{\begin{array}{l}
r_{0}:=\min \left\{n: \alpha_{m}>0, \text { for all } m \geq n\right\} \\
r_{n+1}=\min \left\{i>r_{n}: \alpha_{i}<\alpha_{r_{n}}\right\}
\end{array}\right.
$$

and $\sum_{i}\left(r_{i+1}-r_{i}\right) \alpha_{r_{i}}<+\infty$.

Moreover, if $\alpha_{i}>0$ for all $i \in \mathbb{N}$ then the previous two statements are equivalent to

(c) $\sum_{i} \bar{\alpha}_{i}<+\infty$.

Proof. (a) $\Longrightarrow$ (b). Suppose that (a) holds and there exists $r_{0} \in \mathbb{N}$ such that, for all $n \geq r_{0}$, we have $\alpha_{n}>0$. Suppose that $r_{0}=0$ (the proof in the general case follows easily from this particular case). Then $\lim _{i} \alpha_{n_{i}}=0$ and, since $\alpha_{n_{i}}>0$ for all $i \in \mathbb{N}$, we have that, for all $j \in \mathbb{N}$, the set $\left\{i: \alpha_{n_{i}}<\alpha_{j}\right\} \neq \varnothing$ and it is possible to define recursively the sequence $\left\{r_{n}\right\}$. Clearly, we have

$$
\alpha_{i} \geq \alpha_{r_{n}}, \quad \text { for all } i<r_{n+1} .
$$

We show now that for all increasing sequences $\left\{n_{i}\right\}_{i}$ we have

$$
\sum_{i}\left(n_{i+1}-n_{i}\right) \alpha_{n_{i}} \geq \sum_{i}\left(r_{i+1}-r_{i}\right) \alpha_{r_{i}},
$$

which easily implies (b). Indeed, note that if we define $\gamma_{j}=\alpha_{r_{i}}$ for all $j \in\left[r_{i}, r_{i+1}\right)$, then

$$
\sum_{i}\left(r_{i+1}-r_{i}\right) \alpha_{r_{i}}=\sum_{j} \gamma_{j}
$$

similarly if $\gamma_{j}^{\prime}=\alpha_{n_{i}}$, for all $j \in\left[n_{i}, n_{i+1}\right)$, then

$$
\sum_{i}\left(n_{i+1}-n_{i}\right) \alpha_{n_{i}}=\sum_{j} \gamma_{j}^{\prime}
$$

Let us fix $j \in \mathbb{N}$ and suppose that $j \in\left[r_{i}, r_{i+1}\right) \cap\left[n_{l}, n_{l+1}\right)$, then $n_{l}<r_{i+1}$; whence, (5.8) implies that

$$
\gamma_{j}^{\prime}=\alpha_{n_{l}} \geq \alpha_{r_{i}}=\gamma_{j}
$$

Thus, $\gamma_{j}^{\prime} \geq \gamma_{j}$ for all $j \in \mathbb{N}$. 
(b) $\Longrightarrow$ (a). This part is straightforward.

(b) $\Longrightarrow$ (c). Since $\alpha_{n}>0$ for all $n \in \mathbb{N}$, let us define $\bar{n}_{i}=r_{i}$ and let $\left\{\gamma_{i}\right\}$ be as before. The sequence $\left\{\alpha_{\bar{n}_{i}}\right\}$ is clearly nonincreasing. Using (5.9), we just need to prove that $\gamma_{n}=\bar{\alpha}_{n}$ for all $n$. Indeed, if $n \in\left[\bar{n}_{i}, \bar{n}_{i+1}\right)$, then

$$
\gamma_{n}=\alpha_{r_{i}}=\alpha_{\bar{n}_{i}} \leq \alpha_{j}
$$

for all $j<r_{i+1}=\bar{n}_{i+1}$. Hence, $\gamma_{n}=\alpha_{\bar{n}_{i}}=\min \left\{\alpha_{j}: j \leq n\right\}=\bar{\alpha}_{n}$.

(c) $\Longrightarrow$ (a). This part is straightforward.

\section{Acknowledgements}

The authors are grateful to an anonymous referee for carefully reading the manuscript and for useful suggestions which helped to improve the paper. The authors are also thankful to NUMEC for the logistic support and to Capes-PROEX and Fapesp (09/52379-8) for the financial support during the visits of Fabio Zucca and Daniela Bertacchi to the Universidade de São Paulo.

\section{References}

[1] Alves, O. S. M., Machado, F. P. and Popov, S. Yu. (2002). The shape theorem for the frog model. Ann. Appl. Prob. 12, 533-546.

[2] Alves, O. S. M., Machado, F. P., Popov, S. Yu. and Ravishankar, K. (2001). The shape theorem for the frog model with random initial configuration. Markov Process. Relat. Fields 7, 525-539.

[3] Bertacchi, D. and Zucca, F. (2008). Critical behaviors and critical values of branching random walks on multigraphs. J. Appl. Prob. 45, 481-497.

[4] Bertacchi, D. and Zucca, F. (2009). Approximating critical parameters of branching random walks. J. Appl. Prob. 46, 463-478.

[5] Bertacchi, D. And Zucca, F. (2009). Characterization of critical values of branching random walks on weighted graphs through infinite-type branching processes. J. Statist. Phys. 134, 53-65.

[6] Bertacchi, D. AND Zucca, F. (2012). Recent results on branching random walks. In Statistical Mechanics and Random Walks: Principles, Processes and Applications, Nova Science Publishers, Hauppauge, NY, pp. 289-340.

[7] Fontes, L. R., Machado, F. P. and Sarkar, A. (2004). The critical probability for the frog model is not a monotonic function of the graph. J. Appl. Prob. 41, 292-298.

[8] Gantert, N. And Schmidt, P. (2009). Recurrence for the frog model with drift on $\mathbb{Z}$. Markov Process. Relat. Fields 15 51-58.

[9] Junior, V. V., Machado, F. P. and Zuluaga, M. (2011). Rumor processes on N. J. Appl. Prob. 48, 624-636.

[10] Lebensztayn, E., Machado, F. P. and Martinez, M. Z. (2010). Nonhomogeneous random walk systems on Z. J Appl. Prob. 47, 562-571.

[11] Lebensztayn, É., Machado, F. P. and Popov, S. (2005). An improved upper bound for the critical probability of the frog model on homogeneous trees. J. Statist. Phys. 119, 331-345.

[12] Machado, F. P., Menshikov, M. V. and Popov, S. Yu. (2001). Recurrence and transience of multitype branching random walks. Stoch. Process. Appl. 91, 21-37.

[13] Pemantle, R. (1992). The contact process on trees. Ann. Prob. 20, 2089-2116.

[14] Pemantle, R. and Stacey, A. M. (2001). The branching random walk and contact process on Galton-Watson and nonhomogeneous trees. Ann. Prob. 29, 1563-1590.

[15] Popov, S. YU. (2001). Frogs in random environment. J. Statist. Phys. 102, 191-201.

[16] Popov, S. Yu. (2003). Frogs and some other interacting random walks models. In Discrete Random Walks (Paris, 2003), Association of Discrete Mathematics and Theoretical Computer Science, Nancy, pp. 277-288.

[17] Telcs, A. And Wormald N. C. (1999). Branching and tree indexed random walks on fractals. J. Appl. Prob. 36, 999-1011.

[18] Zucca, F. (2011). Survival, extinction and approximation of discrete-time branching random walks. J. Statist. Phys. 142, 726-753. 